\title{
Blood-brain barrier resealing in neuromyelitis optica occurs independently of astrocyte regeneration
}

\author{
Anne Winkler, ${ }^{1}$ Claudia Wrzos, ${ }^{1}$ Michael Haberl, ${ }^{2}$ Marie-Theres Weil, ${ }^{3,4}$ Ming Gao, ${ }^{5}$ Wiebke Möbius, ${ }^{3,4}$ Francesca Odoardi, ${ }^{2}$ \\ Dietmar R. Thal, ${ }^{6,7}$ Mayland Chang, ${ }^{5}$ Ghislain Opdenakker, ${ }^{8}$ Jeffrey L. Bennett, ${ }^{9}$ Stefan Nessler, ${ }^{1}$ and Christine Stadelmann ${ }^{1}$ \\ IInstitute of Neuropathology and ${ }^{2}$ Institute for Multiple Sclerosis Research and Neuroimmunology, University Medical Center Cöttingen, Göttingen, Germany. ${ }^{3}$ Electron Microscopy Core Unit, Department \\ of Neurogenetics, Max-Planck-Institute of Experimental Medicine, Göttingen, Germany. ${ }^{4}$ Center Nanoscale Microscopy and Molecular Physiology of the Brain (CNMPB), Göttingen, Cermany. ${ }^{5}$ Department \\ of Chemistry and Biochemistry, University of Notre Dame, Notre Dame, Indiana, USA. 'Department of Imaging and Pathology, KU Leuven, and Department of Pathology, UZ Leuven, Leuven, Belgium. \\ 'Laboratory of Neuropathology, Institute of Pathology, Ulm University, Ulm, Germany. ${ }^{8}$ Laboratory of Immunobiology, Department of Microbiology and Immunology, Rega Institute for Medical Research, \\ KU Leuven, Leuven, Belgium. 'Pepartments of Neurology and Ophthalmology, Program in Neuroscience, University of Colorado at Anschutz Medical Campus, Aurora, Colorado, USA.
}

\begin{abstract}
Approximately $80 \%$ of neuromyelitis optica spectrum disorder (NMOSD) patients harbor serum anti-aquaporin-4 autoantibodies targeting astrocytes in the CNS. Crucial for NMOSD lesion initiation is disruption of the blood-brain barrier (BBB), which allows the entrance of Abs and serum complement into the CNS and which is a target for new NMOSD therapies. Astrocytes have important functions in BBB maintenance; however, the influence of their loss and the role of immune cell infiltration on BBB permeability in NMOSD have not yet been investigated. Using an experimental model of targeted NMOSD lesions in rats, we demonstrate that astrocyte destruction coincides with a transient disruption of the BBB and a selective loss of occludin from tight junctions. It is noteworthy that BBB integrity is reestablished before astrocytes repopulate. Rather than persistent astrocyte loss, polymorphonuclear leukocytes (PMNs) are the main mediators of BBB disruption, and their depletion preserves BBB integrity and prevents astrocyte loss. Inhibition of PMN chemoattraction, activation, and proteolytic function reduces lesion size. In summary, our data support a crucial role for PMNs in BBB disruption and NMOSD lesion development, rendering their recruitment and activation promising therapeutic targets.
\end{abstract}

\section{Introduction}

Aquaporin-4-seropositive (AQP4-seropositive) neuromyelitis optica spectrum disorder (NMOSD) is an astrocytopathic and demyelinating CNS autoimmune disease clinically characterized by severe recurrent attacks of optic neuritis and myelitis, resulting in the rapid attack-related development of permanent neurological deficits. Due to clinical similarities NMOSD was historically regarded as a subtype of multiple sclerosis (MS). This changed, however, with the discovery of autoantibodies directed against the water channel AQP4 (AQP4 Abs) that are present in approximately $80 \%$ of the patients (1). In the CNS, AQP4 is highly expressed on astrocytic endfeet in close proximity to brain postcapillary venules, revealing astrocytes as the primary target in NMOSD. Complement- and cell-mediated Ab-dependent astrocyte destruction secondarily results in loss of oligodendrocytes and demyelination $(2,3)$.

The pathogenicity of AQP4 Abs was substantiated in experimental models in which the intracerebral injection of AQP4 Abs combined with a source of complement or the i.v. injection of AQP4 Abs into animals either primed with an encephalitogenic antigen or injected with CNS antigen-specific activated $\mathrm{T}$ cells

Conflict of interest: The authors have declared that no conflict of interest exists. Copyright: () 2021, American Society for Clinical Investigation.

Submitted: July 2, 2020; Accepted: January 6, 2021; Published: March 1, 2021.

Reference information: J Clin Invest. 2021;131(5):e141694.

https://doi.org/10.1172/JCl141694. reproduced the seminal features of the human disease $(2,4-8)$. Moreover, reducing $\mathrm{Ab}$ titers via plasma exchange and immunoadsorption or by blocking the complement cascade, e.g., with the C5 convertase inhibitor eculizumab, are effective treatments for $\operatorname{NMOSD}(9,10)$.

Although the pathogenicity of AQP4 Abs is widely recognized, little is known about the trigger or triggers of the disease. Systemic application of AQP4 Abs in healthy animals failed to induce NMOSD-like lesions in most studies $(5,11,12)$, except when pathogenic murine monoclonal AQP4 Abs were administered over a prolonged period of time (13). Consistent with these findings, AQP4 seropositivity has been observed in patients well before disease onset (14), indicating that either high titers of pathogenic Abs, local inflammation, or the disruption of the blood-brain barrier (BBB) is necessary for disease induction. Indeed, monoclonal Abs targeting IL-6R have been tested successfully in clinical trials for the treatment of NMOSD (15-17). IL-6, which is increased in the serum and CSF of NMOSD patients, has been shown to directly act on endothelial cells and decrease BBB function (18-20). Interestingly, autoantibodies directed against the endothelial protein GRP78 were identified in NMOSD patients; these may promote $\mathrm{BBB}$ disruption and therefore contribute to lesion initiation and perpetuation in NMOSD patients (21).

The BBB is a selective and protective barrier of the CNS, restricting the diffusion of solutes into the brain and regulating the entry of immune cells. Under inflammatory conditions such as in NMOSD or MS, these functions are disturbed. This is evidenced by 
the presence of immune cell infiltrates in lesions and the leakage of contrast agent into the brain, as seen by MRI. Interestingly, the disruption of the BBB was reported to be more severe in NMOSD than in MS (22). The BBB is formed by specialized brain endothelial cells expressing transporter systems and tight interendothelial junctions, the latter composed of transmembrane proteins, such as occludin, claudin-3, and claudin-5, among others. The endothelial cells closely interact with pericytes and CNS parenchymal cells - predominantly astrocytes - which are important for the maintenance of their BBB phenotype (23). Astrocytes, the cellular targets of the autoimmune response in NMOSD, are lost early during lesion formation, which thus may contribute to the more severe BBB disruption observed (22). On the other hand, activated infiltrating immune cells are the initiators of BBB disruption in experimental models of CNS autoimmunity such as experimental autoimmune encephalomyelitis (EAE) (24). In contrast with what occurs in MS, the immune cell infiltrate in early NMOSD lesions is notably not only composed of macrophages and a few $\mathrm{T}$ and $\mathrm{B}$ cells, but also of polymorphonuclear leukocytes (PMNs) (25).

In the present study, we investigated the time course and mechanisms of BBB breakdown in NMOSD and characterized its cellular, structural, and molecular correlates. We hypothesized that both the infiltration of immune cells and astrocyte loss substantially contribute to BBB disruption in the disease. Our studies in experimental and human NMOSD lesions revealed a transient and short-lived disruption of the BBB mediated by infiltrating PMNs. Interestingly, the permeability of the BBB to i.v. injected tracer molecules was restored in the absence of the tight junction molecule occludin and long before astrocytes repopulated the experimental lesions. Moreover, depletion of PMNs from the blood completely inhibited the development of astrocyte-depleted lesions, and selective inhibition of PMN chemoattraction, activation, and protease function reduced astrocyte lesion size. Also, in human tissue, $P M N$ infiltration correlated with the time point of most intense BBB leakage, and restoration of BBB integrity to fibrinogen was observed in the absence of astrocytes, underlining the role of PMNs for BBB disruption and NMOSD lesion development.

\section{Results}

Disruption of the BBB coincides with lesion development. To elucidate the role of immune cells and astrocytes for BBB permeability in inflammatory astrocyte-depleted lesions, we applied a rat model of focal NMOSD lesions reproducing key features of the human disease, such as loss of astrocytes and oligodendrocytes, demyelination, and immune cell infiltration. It is based on the stereotactic injection of a recombinant human AQP4 Abs together with human complement into the rat cortex, leading to an Ab-mediated lysis of astrocytes (2). To investigate lesion development and BBB disruption, time-course studies were performed. Three hours after lesion induction, single dying glial fibrillary acidic proteinpositive (GFAP-positive) astrocytes were observed (Figure 1A). Confluent astrocyte-depleted lesions developed 6 hours after lesion induction and reached their maximum extent between 24 hours and 3 days. Astrocyte loss was confirmed using the astrocytic marker proteins GFAP, AQP4, S100 $\beta$, EAAT2, ALDH1L1, and SOX9 (Figure 1, B and C, and Supplemental Figure 1; supplemental material available online with this article; https://doi.
org/10.1172/JCI141694DS1). Subsequent astrocyte repopulation led to substantial lesion repair by day 7 (Figure 1D) and complete repopulation 14 days after lesion induction (2). Control animals intracerebrally injected with a non-CNS-specific anti-measles control $\mathrm{Ab}$ and human complement did not develop astrocytedepleted lesions (Figure 1D).

To analyze the time course of $\mathrm{BBB}$ permeability in rats induced with focal NMOSD lesions, endogenous as well as i.v. injected tracer molecules were investigated. Six hours after lesion induction, the plasma proteins IgG $(150 \mathrm{kDa})$ and fibrinogen $(340 \mathrm{kDa})$ were observed perivascularly in the brain parenchyma at the injection site, accumulated until 24 hours, and were largely removed after 3 days (Supplemental Figure 2). Similarly, the lysine-fixable tracer molecules FITC-albumin (70 kDa) and Texas Red cadaverine (690 Da), injected 40 or 50 minutes before perfusion, respectively, penetrated into the parenchyma (Figure 1, E and F). However, no leakage was observed 3 hours after lesion induction, indicating that the injection alone was not causing a detectable disruption of the BBB. Surprisingly, the integrity of the $\mathrm{BBB}$ to both tracers was restored within 24 hours after lesion induction, although the maximum extent of astrocyte loss was measured at this time point (Figure 1, G-J).

Transient loss of occludin from blood vessels in astrocyte-depleted areas. Tight junctions, located at the cell-to-cell contacts of neighboring endothelial cells, prevent the paracellular diffusion of blood-borne molecules into the CNS. In the brain vasculature, the transmembrane tight junction proteins occludin, claudin-3, and claudin- 5 are highly expressed, and a disruption of their structure or loss of expression has been implicated in the dysfunction of the BBB (26-30). Since we observed a transient loss of BBB integrity in experimental NMOSD lesions, we investigated a possible correlation between the breakdown of the BBB and alterations in the expression of these tight junction molecules. The presence of occludin, claudin-3, or claudin- 5 at the capillaries, visualized by the basal lamina marker LAM $\gamma 1$, was analyzed at various time points after lesion induction in GFAP-depleted areas in antiAQP4 Ab-injected animals. In naive, uninjected controls, sharply defined occludin, claudin-3, and claudin-5 immunoreactivity marks the paracellular cleft of adjacent endothelial cells within the LAM $\gamma 1$-immunopositive basal membrane (Figure 2, A-C). The percentage of blood vessels with localization of claudin-3 and claudin- 5 at the tight junctions did not differ at 6 hours, 10 hours, 24 hours, 3 days, or 6 days (Figure 2, B, C, E, and F) after NMOSD lesion induction. However, a transient loss of occludin immunoreactivity from the blood vessels within astrocyte-depleted lesions was observed starting 6 hours after lesion induction (occludin-positive blood vessels: $93.3 \% \pm 3.3 \%$ in naive controls compared with $30.6 \% \pm 10.1 \% 6$ hours post injection [p.i.]) and reaching the maximum extent at 10 and 24 hours (10 hours: $3.5 \%$ $\pm 1.3 \%$; 24 hours: $3.4 \% \pm 2.0 \%$ ). Subsequently, occludin expression was restored to normal levels within 6 days (3 days p.i.: $68.5 \%$ $\pm 2.3 \%$; 6 days p.i.: $94.6 \% \pm 1.7 \%$; Figure 2 , A and D).

In summary, astrocyte-depleted lesions started to develop 6 hours after lesion induction, accompanied by a simultaneous permeability of the BBB to differently sized tracer molecules. Loss of occludin from blood vessels was observed to begin at the same time point. However, the integrity of the BBB to the tracer mole- 

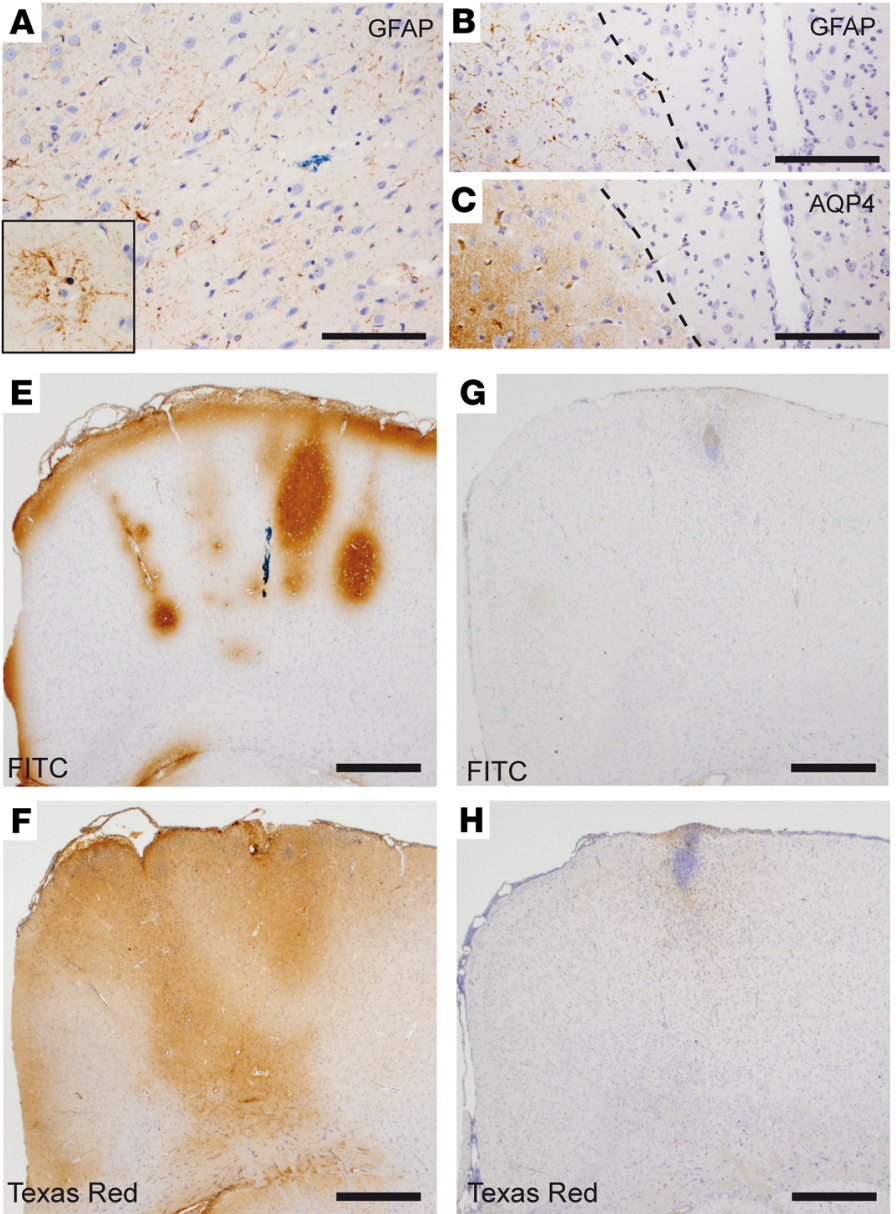

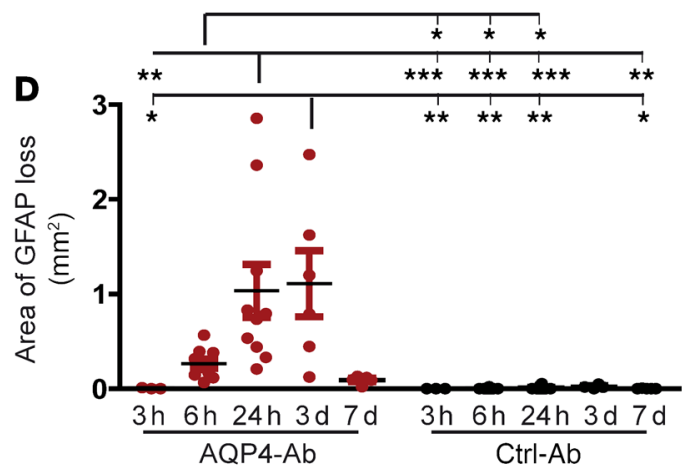

I
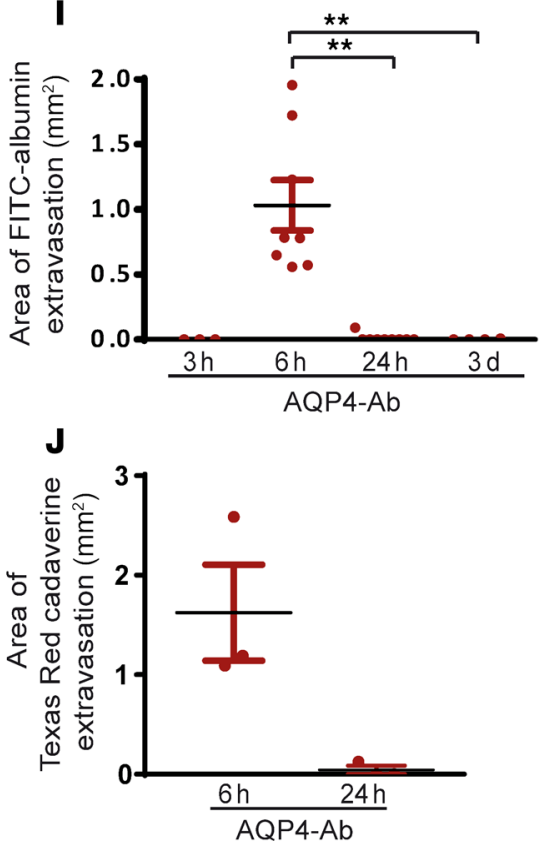

Figure 1. Early loss of astrocytes coincides with BBB breakdown in experimental NMOSD lesions. To assess the time course of astrocyte lesion development, AQP4 Abs and human complement were injected intracortically and animals were perfused after 3 hours, 6 hours, 24 hours, 3 days, and 7 days. Controls were injected with an irrelevant $A b$ in the presence of human complement. Three hours after $A b$ and complement injection, GFAP-positive astrocytes were still observed at the injection site (A). Monastral blue marks the injection site. However, dying GFAP-positive cells with retracting processes were also found (A, insert). Twenty-four hours after injection, large, well-demarcated areas with loss of GFAP (B) and AQP4 immunoreactivity (C; serial sections of the same lesion, astrocyte loss marked by dotted line) were detected. Quantification of GFAP immunoreactivity revealed initial loss 6 hours after lesion induction, peaking between 24 hours and 3 days, with a subsequent repopulation of GFAP-positive cells. No astrocyte loss is observed after injection of control Ab (ctrl-Ab) together with human complement. Number of lesions: 3 hours, $n=3 ; 6$ hours and 24 hours, $n=10 ; 3$ days, AQP4/2B4, $n=6 / 4 ; 7$ days, $n=5$ (D). Simultaneously, with the loss of astrocytes, a prominent extravasation of the injected tracers FITC-albumin (60 kDa) (E) and Texas Red cadaverine $(0,69 \mathrm{kDa})(\mathbf{F})$ into the brain parenchyma was observed 6 hours after focal injection. No vascular leakage of either molecule was detected 24 hours after stereotactic injection (G, FITC-albumin; H, Texas Red cadaverine), which is confirmed by quantification (I). Number of lesions: FITC-albumin: 3 hours, $n=3$; 6 hours, $n=8 ; 24$ hours, $n=9 ; 3$ days, $n=4$. (J) Texas Red cadaverine, $n=3$. (D, I, and J) Kruskal-Wallis test followed by Dunn's multiple comparison test. ${ }^{*} P<0.05 ;{ }^{*} P<0.01 ;{ }^{* *} P<0.001$. Graphs are shown as mean \pm SEM. Scale bars: $100 \mu \mathrm{m}(\mathbf{A}-\mathbf{C}) ; 500 \mu \mathrm{m}(\mathbf{E}-\mathbf{H})$.

cules FITC-albumin and Texas Red cadaverine was fully restored in the absence of astrocytes and occludin (Figure 2G), indicating BBB-resealing mechanisms independent of astrocyte contact.

Depletion of immune cells prevents $B B B$ disruption and astrocyte lesion formation. The interaction of inflammatory cells with the activated endothelium results in their transmigration into the CNS, and it can also induce the disruption of the BBB to solutes $(24,31,32)$. Therefore, we determined whether inflammatory cells might be central to the induction of BBB leakiness in early NMOSD lesions by performing depletion studies with immune cell-depleting serum. Depleting serum was injected i.p. 18 hours prior to and directly after intracerebral injection of $\mathrm{AQP} 4 \mathrm{Abs}$ and human complement while control rats received control, nonimmune serum. Treatment with immune cell-depleting serum reduced the levels of all immune cell subsets (PMN numbers by 99.2\%, CD4-positive T cells by $93.5 \%$, CD 45R-positive B cells by $91.9 \%$, and CD11b-positive myeloid cells by $94 \%$ compared with animals treated with control serum; Supplemental Figure 3A). The depletion of inflammatory cells from the blood was also reflected in the brain parenchyma where high numbers of infiltrated immune cells were observed 6 hours after lesion induction, but were absent in the parenchyma of rats treated with depleting serum (Supple- 

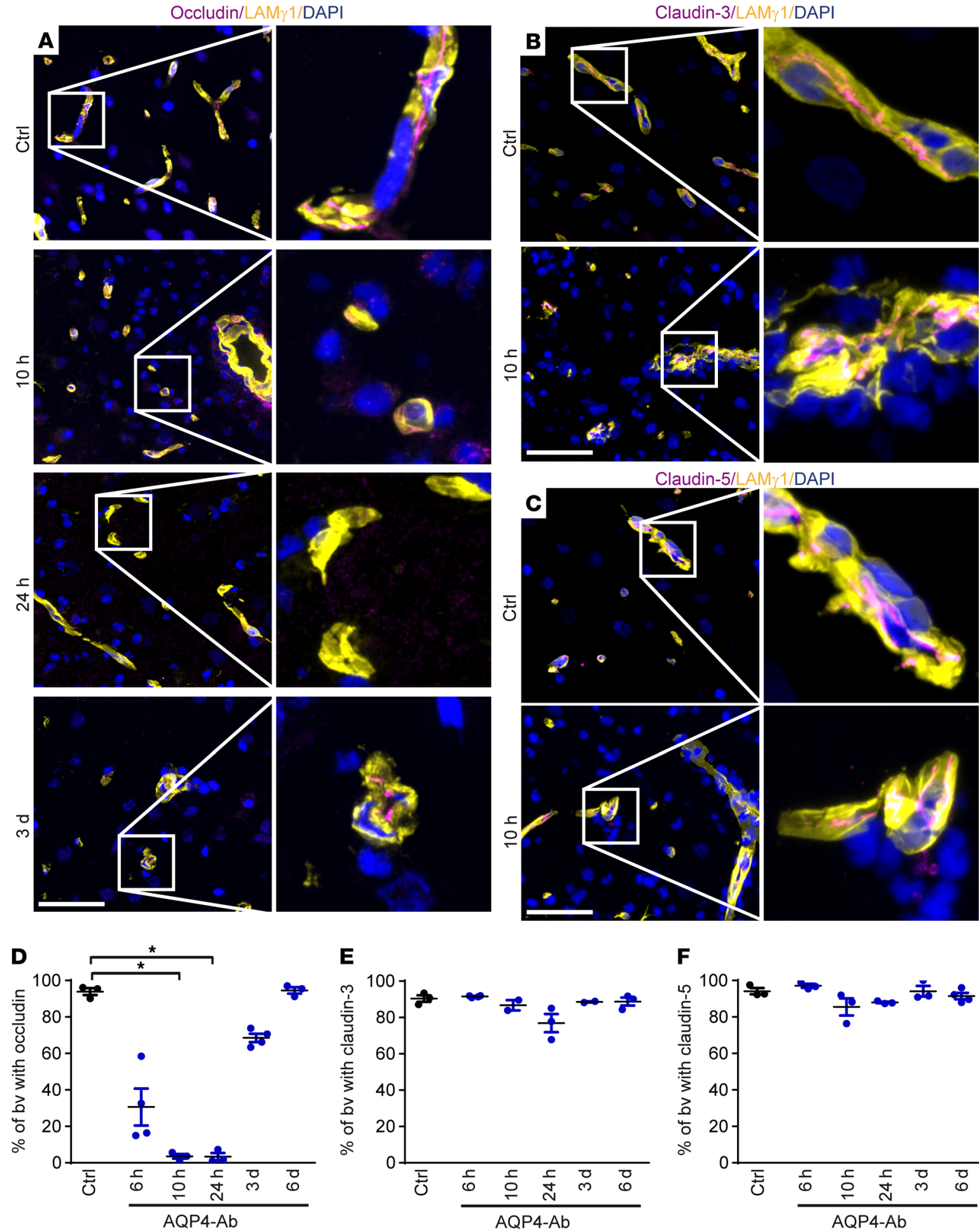

E

\section{$\mathbf{F}$}
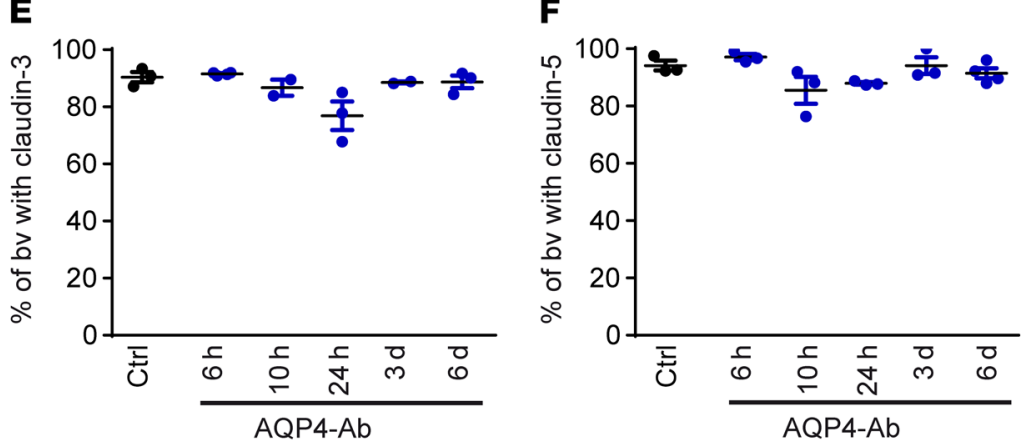

\section{G}

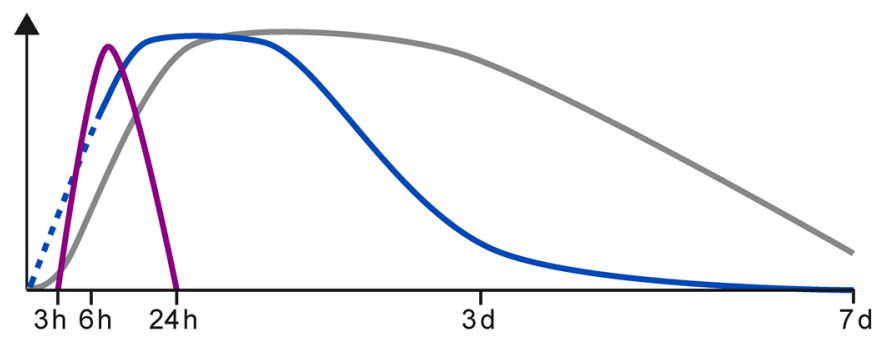

Tracer extravasation

Astrocyte lesion size

Loss of occludin from

blood vessels 
Figure 2. Breakdown of the BBB is accompanied by loss of occludin from blood vessels in experimental NMOSD lesions. Immunolabeling for occludin, claudin-3, and claudin-5 (magenta) was performed to assess the localization of these tight junction proteins in brain vessels (visualized with the basal lamina LAM 1 marker, yellow) in focal NMOSD-like lesions. Immunoreactivity for occludin (A), claudin-3 (B), and claudin-5 (C) was localized at the tight junctions between adjacent endothelial cells and formed sharply defined, continuous strands in uninjected, naive controls. Loss of occludin immunoreactivity was observed 10 hours and 24 hours after lesion induction in astrocyte-depleted areas. Vascular occludin localization recovered to $68.5 \% \pm 4.6 \%$ within 3 days after focal injection of $A Q P 4 A b$ and human complement (A). Quantification of occludin-positive vessels confirms the transient loss of occludin immunoreactivity from blood vessels in areas of GFAP loss (41-307 vessels/animal evaluated depending on astrocyte lesion size; $n=3$ animals per time point, except 6 hours $n=4 ; \mathbf{D}$ ). In contrast, no loss of claudin-3 and claudin- 5 immunoreactivity was detected after lesion induction, and sharply defined immunopositive strands were observed at the tight junctions of LAM $\gamma 1$-positive vessels at 10 hours (B and $\mathbf{C}$, respectively). Quantification of claudin-3-positive vessels (45-218 vessels/animal evaluated; ctrl $n=3$, 6h: $n=4$, 10h: $n=2$, 24h: $n=3$, 3d: $n=2,6 \mathrm{~d}: n=$ 3; E) and claudin-5-positive vessels (28-209 vessels/animal, $n=3$ animals per time point, except $6 h n=4 ; \mathbf{F}$ ) confirms this observation. Temporal evolution of experimental NMOSD lesions (G). The $y$ axis represents the extent of the investigated factors in arbitrary units. (D-F) Kruskal-Wallis test followed by Dunn's multiple comparison test. ${ }^{*} P<0.05$. Data are shown as mean \pm SEM. bv, blood vessels. Scale bars: $50 \mu \mathrm{m}$ (A-C).

mental Figure 3B). Moreover, immune cell depletion resulted in the preservation of BBB integrity to FITC-albumin, which was injected i.v. 40 minutes before perfusion (Supplemental Figure 3C). Correspondingly, there was no loss of occludin from the tight junctions of these animals (Supplemental Figure 3D). However, not only BBB disruption, but also astrocyte loss, was completely prevented by immune cell depletion. (Supplemental Figure 3E).

$P M N$ s are the first immune cells to infiltrate experimental NMOSD lesions, their numbers correlate with FITC-albumin extravasation, and specific depletion halts NMOSD lesion development. To more specifically identify the immune cells responsible for BBB disruption, immune cell subsets were analyzed in experimental NMOSD lesions at various time points (Figure 3, A and B). Abundant infiltrating PMNs, visualized by chloroacetate esterase (CAE) enzyme histochemistry, were already observed in the brain parenchyma 6 hours after lesion induction and reached their maximum density at the injection site between 12 hours and 24 hours. In contrast, few macrophages/activated microglia, stained by ED1 immunohistochemistry, infiltrated at the injection site 6 hours after lesion induction. Their density subsequently increased, reaching a peak 24 hours after lesion induction. Immunohistochemistry for CD3 showed that $\mathrm{T}$ cell numbers remained low in the CNS at all times investigated. Six hours after lesion induction - a time concurrent with the maximum disruption of the $\mathrm{BBB}$ and the development of confluent astrocyte-depleted lesions - the density of PMNs/ $\mathrm{mm}^{2}$ was significantly higher compared with that of ED1- or CD3positive cells (Figure 3C). Therefore, we investigated a possible relationship between PMN infiltration and BBB permeability in NMOSD lesions. The total number of parenchymal PMNs around the injection site was determined and correlated with the area of FITC-albumin extravasation in serial sections 6 hours after lesion induction. Both parameters were highly correlated, warranting the hypothesis that PMNs might be instrumental in the regulation of BBB permeability during the initiation of experimental NMOSD lesions (Figure 3D).

To address this hypothesis, we performed selective PMN depletion studies using the mAb RP-3, which was applied i.p. 18 hours and briefly before lesion induction. Treatment with RP-3 resulted in a decrease in PMN blood counts to $19.5 \% \pm 7 \%$ (mean \pm SEM) compared with that in animals injected with an isotype control Ab, while monocyte numbers remained stable (154.8\% $\pm 67 \%$ compared with controls, not significant) and lymphocyte numbers significantly increased after RP-3 treatment $(200.4 \% \pm 28.1 \%$ compared with controls, $P=0.04)$. The strong reduction in blood PMN numbers resulted in a significant decrease in extravasated PMNs 6 hours after lesion induction (Figure 4A). This not only significantly reduced BBB disruption, but also astrocyte loss (Figure 4, B and C). These results demonstrate that local stereotactic injection of AQP4 Abs and human complement alone is not sufficient to induce the development of experimental NMOSD lesions in our rat model. Rather, PMN infiltration and the associated disruption of the $\mathrm{BBB}$ that allows, e.g., ample access of complement components and other serum molecules to the CNS appears to be crucially required for astrocyte depletion.

Blockage of complement C5aR decreases astrocyte loss. In a next step, we investigated how PMNs are recruited into nascent experimental NMOSD lesions and whether we might be able to interfere with $\mathrm{BBB}$ disruption and NMOSD lesion formation. We had previously demonstrated that $\mathrm{Ab}$-dependent cell-mediated cytotoxicity as well as complement-dependent cytotoxicity contribute to the formation of astrocyte-depleted lesions in focal experimental NMOSD (2). The anaphylatoxin component 5a (C5a) is a potent PMN chemoattractant and activator that is released during activation of the complement cascade, initiated in this case by the binding of anti-AQP4 Abs to their target. To determine whether C5a-mediated PMN recruitment and activation contribute to BBB disruption and the formation of experimental NMOSD lesions, rats were i.v. injected with the small molecule C5a receptor (C5aR) antagonist PMX-53 at 3 hours after lesion induction. By systemic blocking of C5aR-mediated signaling, astrocyte lesion development was significantly reduced (Supplemental Figure 4A). However, although the number of extravasated PMNs in the lesions was slightly reduced in PMX-53-treated animals, the effect was not significant (Supplemental Figure 4B), and no difference was observed in BBB permeability among treatment groups (Supplemental Figure 4C). This indicates that the anaphylatoxin C5a is not the only mediator of PMN attraction.

Inhibition of PMN-released proteases decreases lesion size. Preformed proteases released from the granules of PMNs upon activation represent another pathway by which PMNs contribute to CNS tissue damage and increased BBB permeability. Two of these proteases are MMP-9 and elastase (33-35). We thus investigated their direct effect on endothelial cells in vitro and assessed their function in experimental NMOSD lesion development in vivo. The direct influence of PMN-derived proteases on $\mathrm{BBB}$ integrity was examined using primary rat brain endothelial cell monolayers seeded in the apical compartment of a Transwell system (Supplemental Figure 5A). Treatment of endothelial monolayers with MMP-9, but not elastase, reduced transendothelial 
A
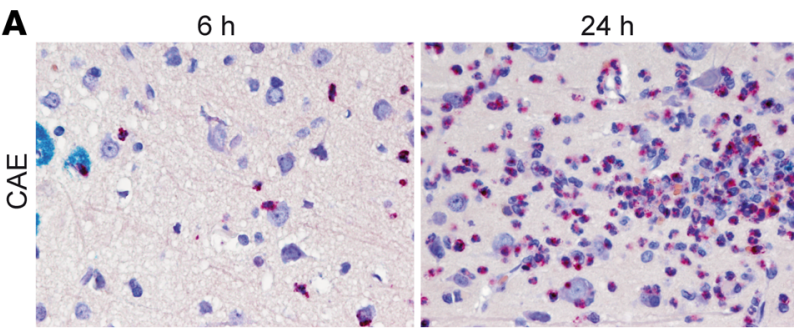

$3 d$

$7 d$
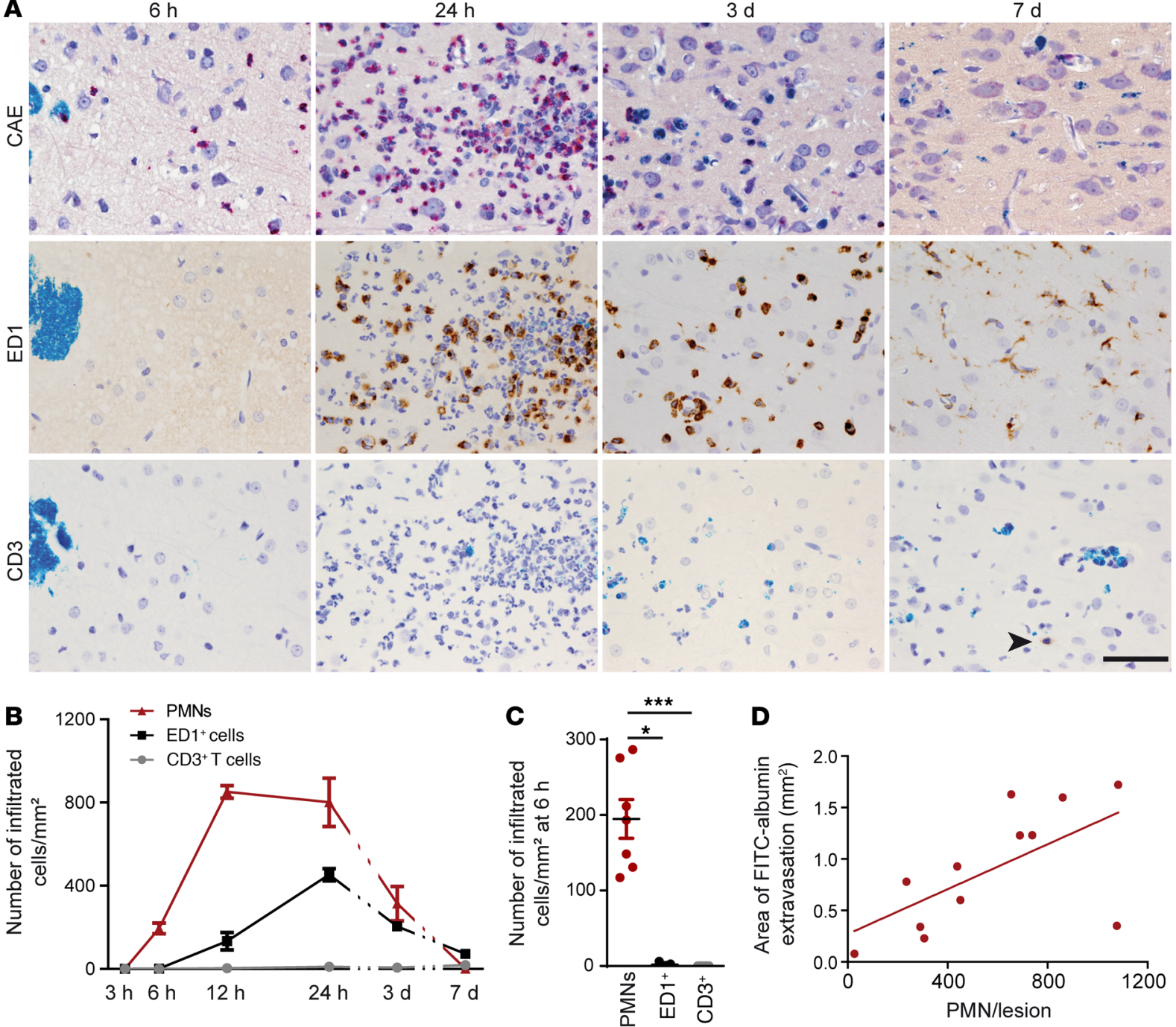

Figure 3. Infiltration of PMNs correlates with extravasation of FITC-albumin 6 hours after lesion induction. Representative photographs depicting the infiltration of PMNs (CAE, pink), macrophages/activated microglia (ED1, brown) and T cells (CD3, brown, arrowhead) 6 hours, 24 hours, 3 days, and 7 days after lesion induction (marked by Monastral blue) (A). Quantification of the infiltration shows the absence of immune cells in the brain parenchyma 3 hours after lesion induction. Six hours after lesion induction, infiltration of PMNs was observed; it reached a maximal density at between 12 and 24 hours and then subsequently decreased. ED1-positive macrophages/activated microglia begin to infiltrate the parenchyma at low numbers 6 hours after lesion induction and reached their highest density at 24 hours. Only a few CD3-positive T cells were found during the time course. Number of lesions analyzed: $n=4,3$ hours, 12 hours; $n=7,6$ hours, 24 hours; $n=6,3$ days, 7 days (B). PMNs had the highest density of all infiltrated immune cell subsets 6 hours after lesion induction (C). The number of infiltrated PMNs at 6 hours correlates with the area of FITC-albumin extravasation into the brain parenchyma. Pearson's correlation for normally distributed samples. Pearson's correlation for normally distributed samples, $P=0,0291 ;$ Pearson's $r=0.6271, n=12$ (D). (C) Kruskal-Wallis test followed by Dunn's multiple comparison test. ${ }^{*} P<0.05 ;{ }^{* *} P<0.001$. Data are shown as mean \pm SEM. Scale bar: $50 \mu \mathrm{m}$.

resistance (Supplemental Figure 5B). This was not mediated by a cytotoxic effect on endothelial cells, as confirmed by lactate dehydrogenase (LDH) assay (Supplemental Figure 5C). In vivo, $19.3 \% \pm 5.4 \%$ of the infiltrating PMNs stained positive for MMP-9 at 6 hours after lesion induction, which decreased to $1.7 \% \pm 0.7 \%$ after 24 hours, indicating a release of MMP-9 early on during lesion formation (Figure 5, A-C). Consequently, we systemically blocked MMP-9 activity in vivo by using the MMP-9 inhibitor ND-336, which is a mechanism-based slow-binding inhibitor of MMP-9 $\left(K_{\mathrm{i}}=150 \pm 10 \mathrm{nM}\right), \mathrm{MMP}-2\left(K_{\mathrm{i}}=85 \pm 1 \mathrm{nM}\right)$, and MMP$14\left(K_{\mathrm{i}}=120 \pm 10 \mathrm{nM}\right)(36)$. ND-336 was injected briefly before the stereotactic injection as well as 2 hours later. We then investi- gated NMOSD lesion development and BBB disruption. Indeed, blocking of MMP-9 significantly decreased the area of astrocyte loss compared with that of vehicle-treated controls (Figure 5D). Although a trend toward decreased infiltration of PMNs and extravasation of FITC-albumin was observed, neither reached significance, indicating that proteolytic functions participating in PMN transmigration also operate in redundant pathways (Figure 5, E and F, respectively). Since ND-336 inhibits both MMP-2 and MMP-14 function, we cannot exclude a participation of either protease in NMOSD lesion development.

Neutrophil elastase (NE) released from PMNs cleaves ICAM-1 and may therefore be important for PMN extravasation from the 

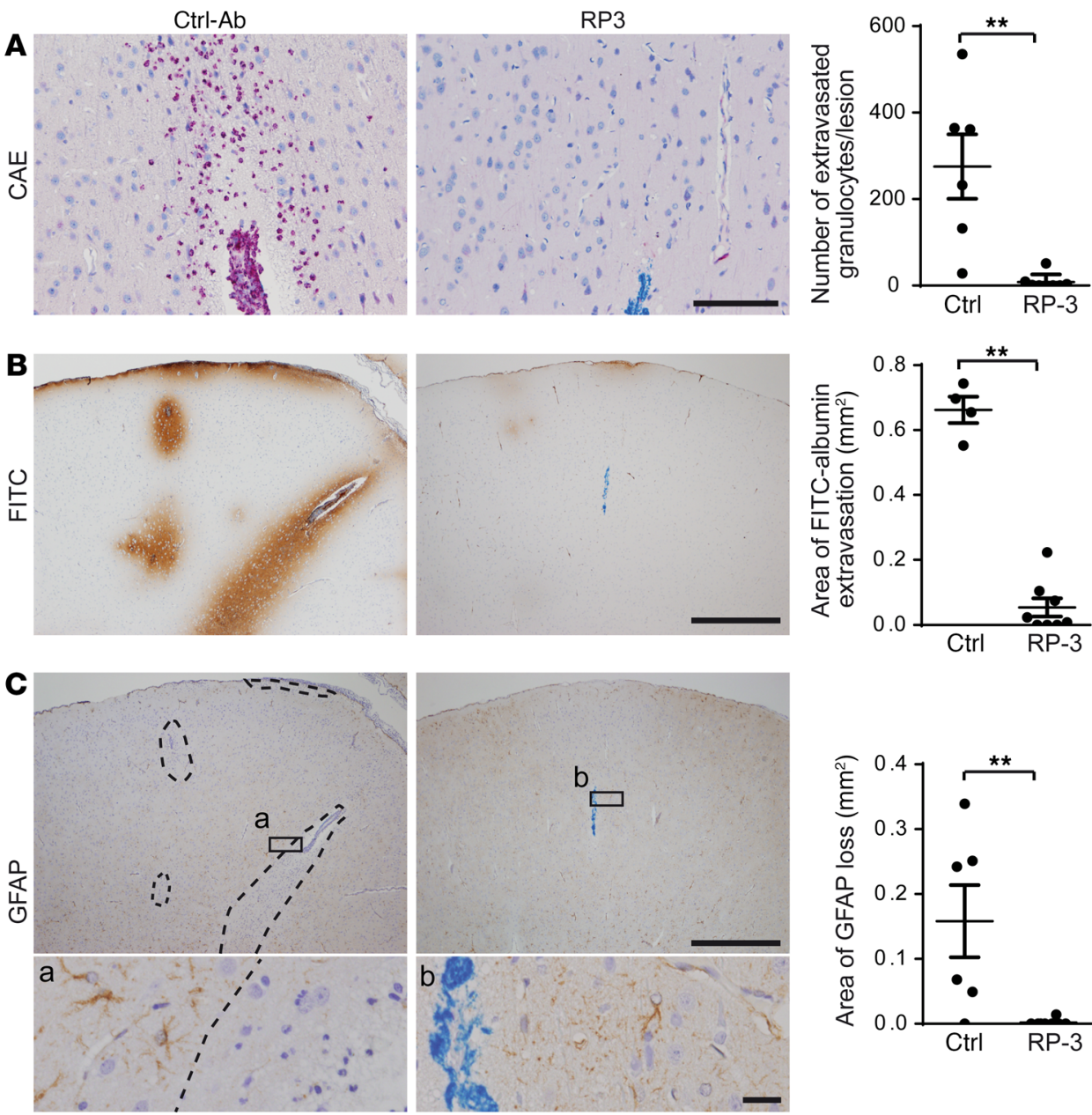

blood $(37,38)$. Moreover, elastase has been shown to exacerbate CNS tissue damage in NMOSD (33). Therefore, we studied the effect of elastase on NMOSD lesion development in vivo. The competitive elastase inhibitor sivelestat was injected i.p. 3 hours after lesion induction, and animals were sacrificed at 6 hours. Inhibition of elastase resulted in significantly reduced astrocyte loss and reduced numbers of infiltrating PMNs (Figure 5, G and $\mathrm{H}$, respectively), compatible with the notion that transmigration is impaired and Ab-dependent cell-mediated cytotoxicity of astrocytes reduced. However, no difference was detected in the extent of BBB disruption (Figure 5I), which corresponded to the finding that even reduced numbers of transmigrating activated PMNs are able to cause substantial BBB leakiness.

Focal loss of astrocytes, disruption of the $B B B$, and immune cell infiltration coincide in human NMOSD lesions. To investigate whether our findings in the focal NMOSD model are mirrored in human NMOSD lesions, biopsy and autopsy tissue of 10 patients with NMOSD was analyzed (Table 1). Early NMOSD lesions showed a distinctive loss of astrocytes and a variable degree of demyelination (Supplemental Figure 6, A-C). Apart from abundant KiM1Ppositive foamy macrophages/activated microglia, myeloid related protein 14-positive (MRP14-positive) recently invaded monocytes/PMNs, scattered T cells and - as in the focal NMOSD model - PMNs were observed (Supplemental Figure 6, D-I).
Figure 4. Depletion of PMNs prevents BBB disruption and astrocyte lesion formation 6 hours after lesion induction. The mAb RP-3 was injected i.p. 18 hours prior to and at the time of lesion induction, and animals were perfused 6 hours later. Treatment of rats with RP-3 specifically decreased blood PMN numbers to $10 \%$ compared with control Ab-injected animals, which is reflected by a significant reduction in PMN numbers in the brain parenchyma 6 hours after lesion induction (A). PMNs are shown as pink using CAE stain; number of lesions: $n=6$. Depletion of PMNs resulted in a significant reduction in FITC-albumin extravasation (B). Number of lesions: control, $n=4$; RP-3, $n=6$. Astrocyte lesion formation (C, dotted lines indicate area of GFAP loss, higher magnification of insets shown in a and $b$; number of lesions $n=6$ ). MannWhitney $U$ test. ${ }^{*} P<0.01$. Data are shown as mean \pm SEM. Scale bars: $100 \mu \mathrm{m}$ (A); $500 \mu \mathrm{m}$ (B and C); $20 \mu \mathrm{m}$ (a and b).

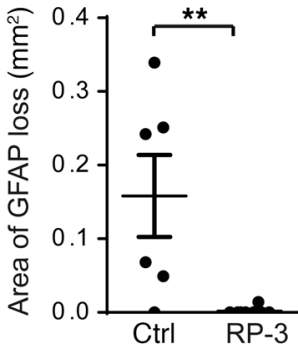

To analyze BBB disruption in NMOSD lesions, fibrinogen extravasation was investigated. In a very early biopsy lesion with ongoing astrocyte lysis, fibrinogen was present around blood vessels (Figure 6A). Within the lesion, $46.4 \mathrm{PMNs} / \mathrm{mm}^{2}$ were counted. However, no perivascular fibrinogen deposits were detected in later stage astrocyte-depleted and not yet repopulated areas of 1 biopsy and 4 autopsy lesions where no infiltration with PMNs and more scattered MRP14-positive monocytes were observed (biopsy: O PMNs/ $\mathrm{mm}^{2}$, autopsies: $8 \pm 5.9 \mathrm{PMNs} / \mathrm{mm}^{2}$ [mean \pm SEM]) (Figure $6, \mathrm{~B}$ and $\mathrm{C}$ ). To study the structural correlates that restrict the paracellular diffusion of solutes, we additionally investigated the expression of the interendothelial tight junction protein claudin- 5 in collagen IV-positive blood vessels using FFPE tissue of these 4 NMOSD autopsies compared with controls (Table 2). In line with the data obtained in our lesion model, we did not find significant differences in claudin-5 expression in blood vessels between the groups (Figure 6, D and E). Additionally, we analyzed occludin and claudin-3 expression in a cryopreserved subacute spinal NMOSD lesion obtained at autopsy, characterized by partial astrozyte repopulation and the absence of MRP14-positive cells (for histological characterization, see Supplemental Figure 7). We found also that these tight junction molecules were expressed at the interendothelial cleft of blood vessels without perivascular astrocyte endfeet (Figure 6F). Moreover, tight junctions in this case also displayed a 

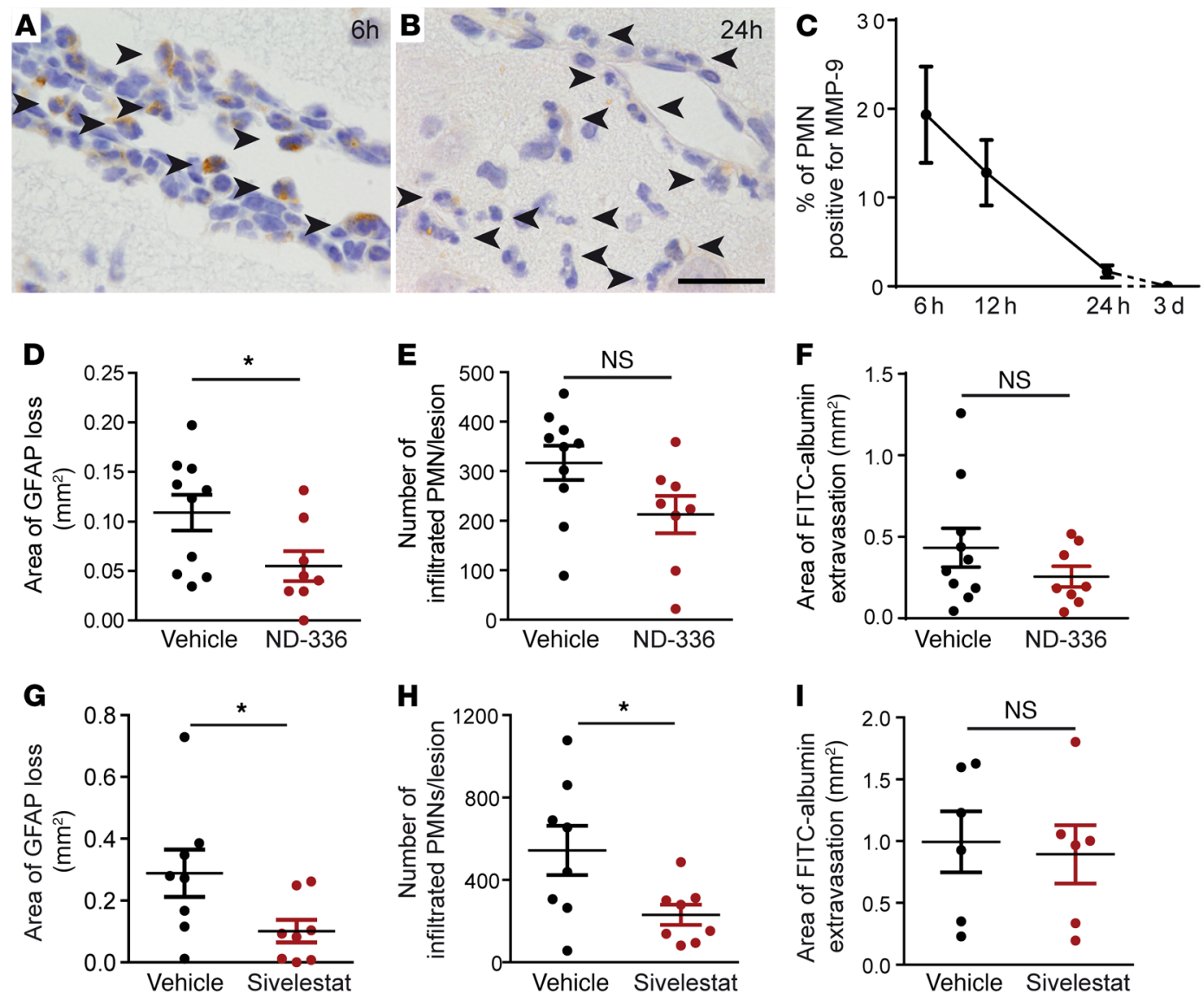

Figure 5. Inhibition of MMP-9 and elastase results in a significant reduction of astrocyte lesion size and PMN infiltration 6 hours after lesion induction. Six hours after lesion induction, numerous vascular and extravasating PMNs display granular intracellular staining for MMP-9 (arrowheads) (A). In contrast, rare MMP-9-positive PMNs are detected 24 hours after lesion induction (B). Quantification of MMP-9-positive PMNs in experimental NMOSD lesions shows a gradual decrease in MMP-9 expression over time, leading to a complete loss of MMP-9 immunoreactivity 3 days after lesion induction (number of lesions: $n=4$, except 3 days, $n=3$ ) (C). To study the function of MMP-9 in NMOSD lesion formation, animals were treated with the selective small molecule MMP-9 inhibitor ND-336, resulting in a significant reduction in astrocyte loss (CFAP) (D) and a trend toward reduced PMN numbers (E) ( $P$ $=0.0595)$ and FITC-albumin extravasation $(\mathbf{F})(P=0.0652)$. Treatment of animals with $50 \mathrm{mg} / \mathrm{kg}$ of the elastase inhibitor sivelestat resulted in a significant reduction in astrocyte loss (GFAP) (G) and PMN infiltration (H). Compared with vehicle-treated animals, there is no alteration in BBB permeability as measured by FITC-albumin extravasation (I). Unpaired $t$ test with Welch's correction. ${ }^{*} P<0.05$. (D-F) Number of lesions: vehicle, $n=10 ;$ ND-336, $n=8$. (G and $\mathbf{H}) n=8$; (I) $n=6$, pooled data of 2 independent experiments. Data are shown as mean \pm SEM. Scale bar: $20 \mu \mathrm{m}$.

regular ultrastructure, as analyzed by electron microscopy (EM) (Figure 6G). This suggests that in human NMOSD lesions, the BBB is also reestablished to large serum molecules before a repopulation with polarized astrocytes has occurred.

\section{Discussion}

Anti-AQP4 seropositive NMOSD is a chronic inflammatory CNS disease in which autoantibodies play a pivotal and tissue-damaging role. Early NMOSD lesions are characterized by astrocyte depletion and inflammatory cell infiltration accompanied by a substantial disruption of the BBB. Increased BBB permeability is a prerequisite for $\mathrm{Ab}$ entry and also allows the passage of complement and other large serum molecules. However, the exact triggers of BBB disruption in NMOSD and the role of astrocyte loss for the observed increase in BBB permeability are not well understood. We demonstrate here in a focal rat model of NMOSD that PMNs are crucial inductors of BBB permeability and NMOSD lesion formation. Selective blockage of PMN entry and PMN pro- tease activity leads to substantially diminished astrocyte-depleted lesions. However, our data also reveal that the increase in BBB permeability in experimental NMOSD is transient and resolves in the absence of astrocytes.

It is well established that the CNS environment is necessary for the development of "typical" BBB properties, such as the polarized expression of transporters and the development of tight junctions, and that $\mathrm{CNS}$ cells regulate $\mathrm{BBB}$ properties, e.g., during neuroinflammation $(24,39)$. In this context, astrocytes are thought to play a vital role (40), leading to our hypothesis that the loss of astrocytes from NMOSD lesions might correlate with the extent and duration of BBB disruption. Using the endogenous bloodborne molecules $\operatorname{IgG}$ and fibrinogen and the endovascular tracer molecules FITC-albumin (70 kDa) and Texas Red cadaverine $(690 \mathrm{Da})$, we show a transient opening of the BBB starting 6 hours after experimental NMOSD lesion induction. Simultaneously, we observed the development of confluent astrocyte-depleted lesions. However, contrary to our hypothesis, within 24 hours, the 
Table 1. Clinical data of NMOSD patients included in study

\begin{tabular}{|c|c|c|c|c|c|c|c|c|}
\hline Patient no. & $\mathrm{B} / \mathrm{A}$ & Age/sex & $\begin{array}{c}\text { Disease duration } \\
\text { (years) }\end{array}$ & $\begin{array}{l}\text { Disease } \\
\text { course }\end{array}$ & Lesions studied & Staging of investigated lesion & $\begin{array}{c}\text { Serum } \\
\mathrm{AQP4} \mathrm{Ab}^{+}\end{array}$ & $\begin{array}{l}\text { Wingerchuk } \\
\text { criteria fulfilled }\end{array}$ \\
\hline 1 & B & $37 / F$ & 9 & $\mathrm{R}$ & Occipital brain lesion & Early; established astrocyte loss & y & y \\
\hline 2 & $B$ & $42 / \mathrm{M}$ & 10 & $\mathrm{R}$ & Frontal brain lesion & Early; established astrocyte loss & y & y \\
\hline 3 & B & $57 / M$ & 10 & $\mathrm{R}$ & Parietal brain lesion & Very early; ongoing astrocyte lysis & y & y \\
\hline 4 & B & $67 / F$ & $<1$ & NA & Parietal brain lesion & Early; established astrocyte loss & y & NA \\
\hline 5 & $B$ & $45 / F$ & 2 & $\mathrm{R}$ & Spinal lesion & Early; established astrocyte loss & y & y \\
\hline 6 & A & $77 / F$ & 24 & $\mathrm{R}$ & Spinal and hippocampal lesion & Early; established astrocyte loss & y & y \\
\hline 7 & A & $72 / \mathrm{M}$ & 0.75 & $\mathrm{R}$ & Spinal lesion & Subacute; partial astrocyte repopulation & y & y \\
\hline 8 & A & $69 / F$ & 0.4 & $\mathrm{R}$ & Basal ganglia lesion & Early; established astrocyte loss & NA & $N A^{A}$ \\
\hline 9 & A & $44 / F$ & 1.5 & $\mathrm{R}$ & Spinal lesion & Early; established astrocyte loss & NA & y \\
\hline 10 & A & $49 / F$ & 0.3 & $\mathrm{R}$ & Spinal lesion & Early; established astrocyte loss & NA & $N A^{A}$ \\
\hline
\end{tabular}

B, biopsy; A, autopsy; F, female; M, male, $R$, relapsing; $N A=$ not available: $y$, yes. ${ }^{A}$ Fulfillment of at least 2 clinical core characteristics (optic neuritis/acute myelitis/area postrema syndrome).

integrity of the $\mathrm{BBB}$ was restored to both larger and smaller tracer molecules in the absence of astrocytes, while deposits of IgG and fibrinogen remained. Only a few studies have investigated the influence of astrocyte depletion on in vivo BBB function in the adult brain, with contradictory results. One study using a genetic approach showed that ganciclovir-mediated depletion of reactive, GFAP-expressing astrocytes after a forebrain stab injury resulted in a prolonged opening of the BBB, but also in more intense inflammatory cell infiltration (41). Recently, another study found that local caspase-9-induced ablation of GFAP-positive astrocytes did not result in increased BBB permeability (42). In a toxic model of 3-chloropropanediol-mediated astrocyte depletion, the loss of astrocytes was shown to precede BBB disruption. However, BBB integrity was restored to vascular tracers of greater than $10 \mathrm{kDa}$ in the absence of astrocytes $(43,44)$. Similarly, treatment of neonatal rats with $\mathrm{N}$-aminonicotinamine resulted in astrocyte depletion in the brain without BBB disruption (45), while treatment of adult rats depleted astrocytes in the spinal cord, leading to tracer extravasation from some but not all astrocyte-depleted vessels (46). These data, together with our finding that astrocyte loss outlasts BBB disruption in focal NMOSD-like lesions, indicate that a transient loss of astrocytes can be compensated for, thus facilitating rapid resealing of $\mathrm{BBB}$ integrity to solutes.

Fibrinogen - used in this study as a marker of BBB disruption - has been shown to actively promote CNS autoimmunity when extravasating into the brain, where it coagulates into the insoluble fibrin. In EAE, fibrin (ogen) has been demonstrated to activate microglia, resulting in the release of chemokines, antigen presentation, and the recruitment of $\mathrm{T}$ cells and macrophages, aggravating demyelination $(47,48)$. Fibrinogen leakage and microglia activation were the first detectable events of lesion formation in marmoset EAE (49), and both factors were found in "predemyelinating" MS lesions (50). Although fibrinogen has not been studied in NMOSD so far, leakage into the CNS might also here trigger microglia activation and subsequent recruitment of inflammatory cells - including PMNs - and contribute to lesion formation. In future studies, the contribution of fibrinogen to lesion development will, therefore, be crucial to investigate.
Interendothelial tight junctions located at the paracellular cleft are responsible for restricting the diffusion of solutes across the BBB. In experimental NMOSD lesions, we observed a transient loss of occludin from the tight junctions starting 6 hours after lesion induction, which was largely recovered by 3 days. Interestingly, BBB integrity was restored to tracers greater than 690 Da before occludin expression was reestablished at the blood vessels. We did not observe a loss of claudin- 3 or claudin- 5 at any time point investigated. Indeed, claudin- 5 is the only tight junction molecule required for the development of a tight $\mathrm{BBB}$, since mice lacking claudin-5 show a size-selective permeability to vascular tracers of less than $800 \mathrm{Da}$ (51). In contrast, no BBB-specific phenotype was described in mice lacking occludin or claudin-3, although the latter is thought to play a role in the formation of the brain-CSF barrier $(52,53)$. This is supported by in vitro transfection studies of mouse fibroblasts in which claudin, but not occludin expression, induced de novo formation of tight junctions $(54,55)$. Under inflammatory conditions such as in EAE, loss of 1 or multiple tight junction molecules has been observed $(29,56,57)$. More recently, it has been recognized that in EAE, paracellular transmigration of immune cells into the brain leads to tight junction disarrangement, such as the formation of gaps in tight junction strands at the site of diapedesis $(58,59)$. In vitro studies suggest rapid remodelling of tight junctions after paracellular transmigration (57) or, alternatively, a transcellular migration that leaves the tight junctions intact (60). Indeed, in astrocyte-depleted areas of human NMOSD lesions without active astrocyte lysis, inflammatory cell infiltration, and fibrinogen leakage, claudin-5 expression at the tight junctions is similar to that in healthy controls. Additionally, EM analysis of a human NMOSD spinal cord lesion displayed electron-dense, normal-appearing tight junctions, which expressed occludin and claudin- 3 on the immunohistochemical level. One caveat, however, is that the NMOSD lesion available for EM and occludin and claudin-3 immunohistochemistry represents a subacute, later stage NMOSD lesion infiltrated by abundant foam cells, but only scattered MRP14-positive recently invaded monocytes. There, BBB integrity might already be at least largely restored, and deposits of fibrinogen may indicate previous BBB disruption. Nevertheless, these findings indicate that the dis- 

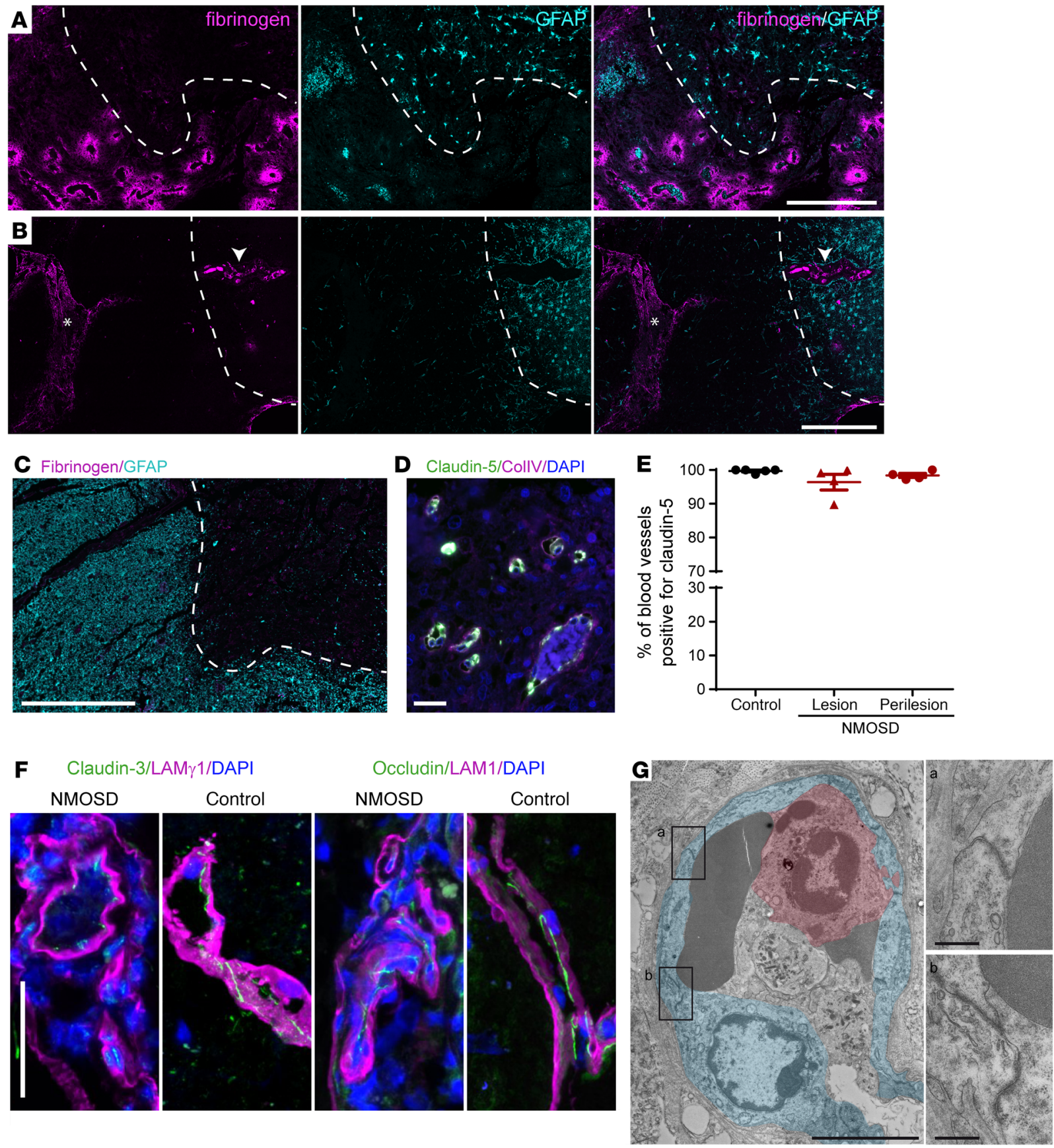

Figure 6. Resealing of the BBB to fibrinogen and tight junctions in human NMOSD lesions. In lesion areas of ongoing and recent astrocyte destruction, perivascular fibrinogen leakage is observed (biopsy, patient 3) (A). No fibrinogen leakage from blood vessels is observed in later-stage human NMOSD lesions with established astrocyte loss (biopsy, patient 1) (B). A resealed BBB was also observed in autopsies with later stage NMOSD lesions (C). Here, claudin-5 expression was present in blood vessels visualized using the basal lamina marker collagen IV (D) (serial section of lesion displayed in C, patient 9). Quantification of the percentage of claudin-5-positive blood vessels is shown (E). Additionally, endothelial cells expressed occludin and claudin-3 at their tight junctions in healthy brain tissue as well as in NMOSD lesions (F). Blood vessels are visualized using the basal lamina marker LAM 1 (patient 7). TJ form electron-dense structures at the intercellular cleft (endothelium, blue; monocyte, red; patient 7). (C). (A-C) Dotted lines delineate lesion border. Asterisks mark bleeding from surgery. Arrowheads indicates big blood vessel. (E) Number of patients: controls, $n=5$; NMOSD, $n=4$. At least 117 blood vessels were evaluated per case. Kruskal-Wallis test followed by Dunn's multiple comparison test revealed no significant differences in the percentage of claudin-5-positive blood vessels between groups. Data are shown as mean \pm SEM. Scale bars: $500 \mu \mathrm{m}$ (A, B, and C); $20 \mu \mathrm{m}$ (D); $50 \mu \mathrm{m}$ (F); $5 \mu \mathrm{m}$ (G); $500 \mathrm{~nm}$ (G, a and b).

ruption of the $\mathrm{BBB}$ can be resolved in the absence of astrocytes. Compensatory mechanisms at the BBB in the absence of astrocytes are also supported by our observation that occludin immunoreactivity is reestablished at the tight junctions in the majority of blood vessels within astrocyte-depleted lesions 3 days after lesion induction. Also, CNS pericytes - which have been shown to be vital for $\mathrm{BBB}$ maintenance $(61,62)$ - may contribute to the rapid stabilization of the BBB in NMOSD, and their role warrants further study. 
Table 2. Clinical data of control patients included in study

$\begin{array}{lcccc}\text { Patient no. } & \text { B/A } & \text { Age/sex } & \text { Cause of death } & \text { Brain area studied } \\ \text { C1 } & \text { A } & \text { 51/F } & \text { Multiorgan failure } & \text { Cervical spinal cord } \\ \text { C2 } & \text { A } & 62 / F & \text { Multi organ failure } & \text { Cervical spinal cord } \\ \text { C3 } & \text { A } & 74 / \mathrm{M} & \text { Not clarified } & \text { Cervical spinal cord } \\ \text { C4 } & \text { A } & 39 / \mathrm{F} & \text { Pulmonary embolism } & \text { Basal ganglia } \\ \text { C5 } & \text { A } & 40 / \mathrm{F} & \text { Not clarified } & \text { Basal ganglia }\end{array}$

Increases in BBB permeability have also been attributed to the infiltration of immune cells into the brain parenchyma. In focal NMOSD-like lesions, we detected only very few CD3-positive T cells, a finding that is in line with previous studies in mice showing that $\mathrm{T}$ cell deficiency does not affect experimental NMOSD lesion development (63) and with the fact that our model is not dependent on immunization and the activation of antigen-specific $\mathrm{T}$ cells. However, abundant infiltrating PMNs, monocytes, and macrophages were observed. Six hours after lesion induction, PMNs were the most abundant leukocytes in the lesions, and importantly, depletion of PMNs prevented astrocyte loss. Indeed, as shown by our group and others, PMNs are a component of the immune cell infiltrate in early human NMOSD lesions $(2,25,64)$ that (actively) participate in NMOSD lesion formation in experimental models. In spinal cord slice cultures, neutrophils induce astrocyte and myelin loss in the presence of sublytic concentrations of AQP $4 \mathrm{Ab}$ and complement (33). In vivo and in line with our experiments, induction of neutropenia in mice resulted in smaller lesions, while neutrophilia exacerbated lesion development (64). Also, it has been demonstrated that PMNs participate in astrocyte damage via Ab-dependent, cellular cytotoxicity mediated by Fc $\gamma$ receptors $(7,65)$.

In addition to direct cytotoxic mechanisms, PMN transmigration into the brain may lead to BBB disruption. This could exacerbate NMOSD lesion formation by facilitating access for further Abs and complement into the brain. Indeed, PMNs were shown to mediate $\mathrm{BBB}$ disruption in various neuroinflammatory models (31, 66-70). In EAE, depletion of PMNs or blockage of CXCR2 - which is important for attracting PMNs - results in the arrest or amelioration of the disease and the preservation of the $\mathrm{BBB} /$ blood-spinal cord barrier $(31,69)$. We demonstrated here a strong correlation between the numbers of infiltrated PMNs and the extent of BBB disruption to FITC-albumin 6 hours after lesion induction. We thus hypothesize that the recruitment of PMNs to early NMOSD lesions may be a key determinant of BBB permeability. Indeed, depletion of PMNs from the blood substantially decreased the disruption of the $\mathrm{BBB}$, as measured using the tracer molecule FITC-albumin.

Our present study aimed at elucidating the pathways by which PMNs are attracted to nascent lesions and participate in NMOSD lesion development. The activation of the complement cascade during AQP4 Ab-mediated astrocyte destruction results in the release of the anaphylatoxin C5a, a potent PMN chemoattractant and activator (71), also in an in vitro NMOSD model (72). In our study, systemic inhibition of C5aR using PMX-53 reduced the area of astrocyte loss in experimental NMOSD lesions. However, we only observed a trend toward reduced PMN infiltration compared with controls and no significant effect on FITCalbumin extravasation. Thus, systemic C5aRblockage might also reduce activation of PMN and their efficiency in Abdependent cell-mediated toxicity. Also, redundant mechanisms for PMN recruitment may play a role. For example, Ab-mediated astrocyte lysis may lead to the local release of chemokines, including chemokines binding to CXCR2, which is highly expressed on PMNs and instrumental in mediating PMN recruitment (73). Another means by which blockage of C5aR may reduce astrocyte lesion size is by inhibiting microglia recruitment and activation (74). Indeed, it has been shown recently that in precytolytic NMOSD lesions, the formation of the anaphylatoxin $\mathrm{C} 3 \mathrm{a}$, which is also released during the activation of the complement cascade, results in astrocyte - microglia crosstalk that may be essential for evolving NMOSD pathology (75).

Additionally, proteolytic enzymes released by PMNs upon activation contribute to tissue damage and $\mathrm{BBB}$ disruption $(76,77)$; this is also true in NMOSD models $(64,65)$. When we focussed on elastase and MMP-9, we observed that MMP-9 reduces transendothelial electrical resistance (TEER) values of primary rat endothelial cells. In vivo, systemic inhibition of either elastase or MMP-9 substantially reduced the area of astrocyte loss in experimental NMOSD lesions 6 hours after lesion induction. While the extravasation of neutrophils into the tissue was markedly impaired upon elastase inhibition, a trend was only observed in animals treated with the MMP-9 inhibitor ND-336. However, inhibition of both MMP-9 and elastase did not result in a better preserved BBB integrity when we used FITC-albumin as a tracer molecule. This notable finding indicates that modulation of PMN function alone, e.g., reduced PMN mobility in the tissue in the case of MMP-9 inhibition, already leads to an impairment of lesion formation. This is the case even in the absence of a reduced BBB permeability to solutes. In addition, our data highlight that a redundant combination of adhesion molecules and PMN-derived proteases contributes to BBB permeability in NMOSD lesions. However, the consistent reduction of NMOSD lesion size in all of our approaches of inhibiting $\mathrm{PMN}$ attraction and effector functions points to the crucial role of PMNs for NMOSD lesion formation.

Our data support a scenario of NMOSD lesion development in which even a few anti-AQP4 Abs binding to their pericapillary target lead to complement activation, PMN recruitment, and an increase in BBB permeability. This may induce a vicious circle leading to expansive focal lesion development. By means of an increase in BBB permeability, PMNs are not only pivotal for Ab-dependent cellular cytotoxicity in NMOSD, but also key regulators of the available complement, thus facilitating complement-dependent cytotoxicity. Blocking PMN entry and PMN-released proteases will thus provide central targets for the development of NMOSD therapies.

\section{Methods}

Human NMOSD tissue. Biopsy and autopsy CNS tissue from 10 NMOSD and 6 control patients was obtained from the archives of the Institute of Neuropathology at the University Medical Center Göttingen. The presence of anti-AQP4 autoantibodies in the serum was confirmed for patients biopsied or autopsied after 2005, but was not 
available for 3 patients who died earlier. Four of five biopsied and three of five autopsied patients fulfilled the revised Wingerchuk criteria of NMOSD (78). Autopsies 8 and 10 were performed in 1962 and 1980, respectively, and no MRI was available. However, patient 8 presented with acute myelitis and area postrema syndrome and patient 10 with acute myelitis and optic neuritis, both therefore fulfilling 2 core characteristics of NMOSD. Neuropathologically, lesions compatible with NMOSD were confirmed by loss of AQP4 and GFAP immunoreactivity in macrophage-rich lesion areas with variable degree of myelin loss. "Very early" NMOSD lesions were characterized by areas of ongoing astrocyte lysis beside astrocyte-depleted areas, the infiltration of abundant MRP14-positive cells (synonyms S100A9, calgranulin B; expressed by both PMNs and recently infiltrated monocytes), relative axonal preservation, and incomplete myelin loss. In later stages of lesion development, "early" NMOSD lesions did not show ongoing astrocyte lysis, harbored less dense $\mathrm{MRP}^{+} 4^{+}$cell infiltrates, and showed more pronounced myelin loss. Frozen spinal cord tissue and tissue suitable for EM was available from 1 patient, autopsy 7, who presented with a "subacute" lesion characterized by partial astrocyte repopulation and abundant $\mathrm{KiM1}^{+}$foamy macrophages, but no $\mathrm{MRP} 4^{+}$cells. For clinical data of NMOSD patients and lesion staging, see Table 1. Autopsy tissue from 5 patients without CNS pathology was used as control (Table 2).

Animals. Adult (180-240 g) female inbred Lewis rats were purchased from Charles River Laboratories. Animals were kept in cages with up to 5 animals each on a 12-hour light/12-hour dark cycle with free access to food and water.

Induction of NMOSD-like lesions in rats. NMOSD-like lesions in rats were induced by intracortical injection of the human recombinant anti-AQP4 Ab 53 (AQP4 Ab), together with human complement. This recombinant monoclonal human IgG1 AQP4 Ab was generated by the paired heavy- and light-chain sequences from a single, CSF-derived, plasma cell clone (4). A similarly produced non-CNS antigen-specific recombinant human IgG1 Ab directed against measles virus nucleocapsid protein (clone rAb 2B4) served as a control (ctrl-Ab). Rats were anesthetized by i.p. injection of ketamine/xylazine. A rostrocaudal cut was performed to gain access to the skull. The head of the animal was fixed in a stereotactic device, and a small hole was drilled through the skull, $1 \mathrm{~mm}$ caudally and $2 \mathrm{~mm}$ laterally to the bregma. Using a finely calibrated glass capillary, $1 \mu \mathrm{l} \mathrm{Ab}$ mixture containing $2.5 \mathrm{mg} /$ $\mathrm{ml} \mathrm{Abs}$ and $15 \mathrm{U} / \mathrm{ml}$ human complement was slowly injected into the cortex. The animals were perfused at various time points after lesion induction ( 3 hours, 6 hours, 12 hours, 24 hours, 3 days, and 7 days). For the assessment of BBB disruption, $1 \mathrm{ml}$ of FITC-albumin (10 mg/ $\mathrm{ml}$, dissolved in saline) or Texas Red cadaverine $(5 \mathrm{mg} / \mathrm{kg}$, dissolved in $4 \% \mathrm{DMSO} /$ saline) was injected 40 minutes or 50 minutes prior to perfusion, respectively.

Depletion studies. For the depletion of immune cells in rats, rabbit anti-PMN antiserum (Accurate Chemical and Scientific Corporation) was injected i.p. at a concentration of $1 \mathrm{ml} / \mathrm{kg}$ body weight (filled to 2 $\mathrm{ml}$ with saline) 18 hours prior to and directly after intracerebral injection of $\mathrm{AQP} 4 \mathrm{Abs}$ and human complement, while control rats received normal rabbit serum (Accurate Chemical and Scientific Corp.). The efficiency of immune cell depletion was analyzed using flow cytometry. Blood samples were diluted 1:1 with PBS. An additional 0.75 volumes of $20 \%$ dextran $(\geq 450 \mathrm{kDa})$ were added and incubated for 30 minutes to allow sedimentation of erythrocytes. The leukocyte-rich plasma was transferred to a new tube and centrifuged at $960 \mathrm{~g}$ for 5 minutes, and the cell pellet was washed once and subsequently resuspended in FACS buffer (2\% BSA, $0.2 \%$ sodium azide in PBS). Prior to neutrophil labeling, cells were fixed with $2 \%$ PFA for 20 minutes, centrifuged, washed with FACS buffer, and centrifuged again. The cells were then permeabilized with $1 \mathrm{BD}$ Perm/Wash buffer. For staining of $\mathrm{CD}^{+} \mathrm{T}$ cells, $\mathrm{CD} 8^{+} \mathrm{T}$ cells, B cells, and myeloid cells, no pretreatment was needed. The following Abs were used (dilution 1:100): PMNs: PE anti-rat granulocytes (clone RP-1, BD Bioscience); CD4 ${ }^{+} \mathrm{T}$ cells: Alexa Fluor 647 anti-rat TCR- $\alpha / \beta$ (clone R73, BioLegend) and PE-Cy5 antirat $\mathrm{CD}^{+}{ }^{+}$(clone OX-35, BD Biosciences); B cells: $\mathrm{PE}$ anti-rat CD45RA (clone OX-33, BioLegend); myeloid cells: Alex Fluor 647 anti-rat CD11b (clone OX-42, AbD Serotec).

To specifically deplete PMNs, rats were injected twice i.p. with 4 mg RP-3 Ab (ref. 79; provided in house) 18 hours prior to and directly before stereotactic injection of AQP4 Abs and human complement. Control animals received control IgM Abs (mouse IgM, clone MM-30, BioLegend) administered by the same treatment regimen. Animals were sacrificed 6 hours after lesion induction, and blood samples were taken. Erythrocytes were lysed using BD FACS Lysing Solution according to the manufacturer's instructions. The PMN depletion efficiency of the RP3 treatment was analyzed by flow cytometry based on the scatter profiles.

Pharmacological inhibition of the complement component 5 a receptor (C5aR), elastase and MMP-9 in vivo. For pharmacological inhibition of C5aR signalling, $1 \mathrm{mg} / \mathrm{kg}$ of the small molecule C5aR antagonist PMX-53 (Teva Pharmaceutical Industries Ltd; diluted to $10 \mathrm{mg} / \mathrm{ml}$ in distilled water and finally to $0.2 \mathrm{mg} / \mathrm{ml}$ in sterile saline $0.9 \%$ ) was i.v. injected 3 hours after NMOSD lesion induction, while control rats were injected with vehicle. 6 hours later rats were perfused and brains were prepared for histological analysis. To inhibit NE, the competitive NE inhibitor sivelestat sodium salt hydrate (R\&D Systems), dissolved to a final concentration of $20 \mathrm{mg} / \mathrm{ml}$ in water, was used. Rats were injected i.p. with $50 \mathrm{mg} / \mathrm{kg}$ body weight sivelestat or vehicle 3 hours after NMOSD lesion induction. They were then perfused 6 hours after intracerebral injection of AQP4 Abs and human complement, and their brains were subsequently processed for histological analysis. To investigate the effect of systemic MMP-9 inactivation on lesion development, the selective MMP-9 inhibitor ND-336 $\left(K_{\mathrm{i}} \mathrm{MMP}-9=150 \mathrm{nM}\right.$, dissolved in $5 \% \mathrm{DMSO} / 20 \%$ propylene glycol/75\% water, synthesized as previously reported; ref. 36) was used; this is able to cross the $\mathrm{BBB}$ at a brain-to-plasma ratio of 0.48 after i.p. and 0.85 after s.c. dosing (our unpublished data). Twice, $25 \mathrm{mg} / \mathrm{kg}$ ND-336 was injected i.p. briefly before the stereotactic injection and then again s.c. 2 hours later. Control rats received vehicle injections only.

Histology and immunohistochemistry. For (immuno-)histochemistry, PFA- and cryo-fixed tissue were used. Brain tissue was analyzed for inflammation, demyelination, and astrocyte loss using H\&E staining, luxol fast blue/periodic-acid Schiff (LFB-PAS) and immunohistochemistry for CD3, CD68, MRP14, AQP4, GFAP, S100 $\beta$, Aldh1L1, EAAT2, and SOX9. The Naphthol AS-D Chloroacetate Esterase Kit (Sigma-Aldrich) was used to visualize PMNs. Disruption of the BBB was visualized using Abs against fibrinogen, IgG, FITC, and Texas Red. Cryopreserved tissue was used for immunohistochemistry of the basal lamina (LAM $\gamma 1$ ) and the tight junction molecules claudin-3, claudin-5, and occludin in experimental NMOSD lesions and a spinal human NMOSD lesion (patient 7). Triple labeling of claudin-5, colla- 
Table 3. Primary Abs

\begin{tabular}{|c|c|c|c|c|c|}
\hline$A b$ & Species & Dilution & Clone number & Product number & Source \\
\hline Aldh1L1 & Mouse & $1: 2000$ & $\mathrm{~N} 103 / 39$ & MABN495 & Merck Millipore \\
\hline AQP4 & Rabbit & 1:100 & - & $A B 2218$ & Merck Millipore \\
\hline CD3 & Rat & $1: 200$ & - & MCA1477 & AbD Serotec \\
\hline CD68 & Mouse & $1: 5000$ & KiM1P & - & (84) \\
\hline Claudin-3 & Rabbit & $1: 100$ & - & AP1S488PU-M & Acris Antibodies Inc. \\
\hline EAAT2 & Guinea pig & $1: 250$ & - & AB1783 & Merck Millipore \\
\hline Fibrinogen & Rabbit & 1:300 & - & A0080 & Dako Deutschland GmbH \\
\hline FITC, HRP conjugated & Rabbit & $1: 50$ & - & P5100 & Dako Deutschland GmbH \\
\hline GFAP & Mouse & 1:300 & 134B1 & 173008 & Synaptic Systems GmbH \\
\hline GFAP & Rat & 1:50 & $2.2 \mathrm{~B} 10$ & $13-0300$ & Thermo Fisher Scientific \\
\hline MRP14 & Mouse & 1:500 & S36.48 & BM4026 & Acris Antibodies Inc. \\
\hline Occludin & Rabbit & $1: 50$ & - & $71-1500$ & Thermo Fisher Scientific, Waltham, MA, USA \\
\hline Immunoglobulin G, biotinylated & Goat & 1:200 & - & RSO00R06 & DCS Innovative Diagnostik-Systeme \\
\hline Texas Red & Rabbit & $1: 200$ & - & A6399 & Life Technologies GmbH \\
\hline
\end{tabular}

gen IV, and GFAP was performed on human FFPE tissue. A list of the primary Abs and their working dilutions is provided in Table 3.

Morphometric analysis and data acquisition. To measure astrocyte lesion size and $\mathrm{BBB}$ opening, images were acquired at $\times 40$ magnification using a BX51 Olympus light microscope equipped with a DP71 digital camera. The images were evaluated using the ImageJ software (80).

For assessment of lesion size, areas of complete loss of AQP4 and GFAP immunoreactivity were measured. For extravasation area, measurements of IgG and fibrinogen, and the tracer molecules FITCalbumin and Texas Red-cadaverine, the brown DAB channel was separated using the ImageJ plugin DAB color deconvolution. Here, the intensity threshold was set to $0-150$ and the resulting marked area was measured. For the IgG and fibrinogen time course, the intensity threshold was adjusted to the staining intensity due to background differences in the different slides.

To evaluate the percentage of blood vessels positive for tight-junction molecules in experimental NMOSD lesions, immunofluorescently labeled sections (claudin-3/claudin-5/occludin, LAM $\gamma 1$, GFAP) were imaged at $\times 100$ magnification using a BX51 Olympus light microscope equipped with a XM10 monochrome camera. The total number of LAM $\gamma 1$-positive blood vessels was counted as well as the number of LAM $\gamma 1$-positive blood vessels immunopositive for occludin, claudin-3, or claudin- 5 at the tight junctions. From this, the percentage of brain vessels with occludin, claudin-3, or claudin-5 localized at the tight junctions was calculated. Similarly, in human NMOSD lesions and healthy control CNS tissue, immunofluorescence triple labeling of claudin-5, collagen IV, and GFAP was used to investigate the percentage of claudin-5-positive blood vessels. For this, immunofluorescently labelled slides were scanned using the VS120 virtual slide microscope (Olympus) at $\times 200$ magnification. Claudin-5-positive and -negative blood vessels were counted using Olympus cellSens Dimension Desk- top software, version 1.16. Here, between 117 and 502 blood vessels were evaluated, depending on the size of the GFAP-depleted area.

To assess the number and density of infiltrating immune cells in human and experimental NMOSD lesions, immunohistochemistry for PMNs and recently infiltrated monocytes/macrophages (MRP14), macrophages/activated microglia (CD68), and T cells (CD3) was performed. In addition, PMNs were identified by CAE enzyme histochemistry (experimental NMOSD) or evaluated based on their multilobular morphology in H\&E (human NMOSD lesions). Cells were counted using a light microscope with an ocular morphometric grid. For assessment of infiltrates in experimental NMOSD lesions, cells were counted in the whole lesion at $\times 100$ magnification. In human patients, the density of immune cells was evaluated by counting the total number of positive cells in 5 representative visual fields at $\times 400$ magnification in astrocyte-depleted very early and early lesion areas with a high density of MRP14-positive recently recruited macrophages and PMNs. In addition, to analyze PMN density in lesion areas with or without BBB disruption, 10 randomly selected visual fields were evaluated in corresponding H\&E-stained sections at $\times 400$ magnification within the lesion. Cell numbers are given as cells $/ \mathrm{mm}^{2}$.

In vitro $B B B$ model. Primary rat brain endothelial cells were isolated based on a modified protocol published by Joan Abbott and coworkers in 2012 (81). Briefly, the brain gray matter of 17-day-old Wistar rats (obtained from the in-house breeding of the Central Department for Animal Experiments, University Medical Center Göttingen) was collected and digested in DMEM containing collagenase D, dispase, and DNase I at $37^{\circ} \mathrm{C}$ for 30 minutes. To separate blood vessels, cells were centrifuged in 20\% BSA/DMEM (1000 g, 20 minutes) after which a microvessel-rich pellet formed at the bottom of the tube. Following an additional digestion time of 20 minutes, the vessel fragments were seeded for 3 days in medium containing puromycin to remove contaminating non-P- 
glycoprotein-expressing cells (82), after which the cells were seeded into Transwell inserts in medium containing hydrocortisone. To assess the integrity of the rat brain endothelial cell monolayer, measurements of TEER were performed, representing the permeability of tight junctions to sodium ions in which high TEER values indicate a tight monolayer. TEER was measured using the EVOM2 resistance meter (World Precision Instruments) with STX-2 electrodes, where one electrode is placed in the apical and the other one in the basolateral compartment. The resistance measured was then expressed relative to the surface area of the insert $\left(\Omega \times \mathrm{cm}^{2}\right)$. TEER values of coated cell-free inserts (140-155 $\Omega$ $x \mathrm{~cm}^{2}$ ) were subtracted from the values of the monolayers. Monolayers were used when they reached on average an electrical resistance of $80 \Omega$ $\times \mathrm{cm}^{2}$. To study the direct influence of the proteases elastase and MMP-9 on the TEER of brain endothelial monolayers, elastase (Merck Millipore, $6 \mu \mathrm{g} / \mathrm{ml}$ ) or active matrix metalloproteinase-9 (Merck Millipore, $0.5 \mu \mathrm{g}$ / $\mathrm{ml}$ ) was added directly to endothelial cells seeded in the apical compartment of a Transwell system. Experiments were performed in triplicate. TEER was measured before treatment and after 60 and 120 minutes. To exclude a direct cytotoxic effect of proteases on brain endothelial cells, the CytoTox-ONE Homogeneous Membrane Integrity Assay (Promega) was used according to the manufacturer's instructions. Measurements were performed after 2 hours of treatment with proteases. Supernatant of lysed RBEC served as positive control.

$E M$. For assessment of the tight junction morphology on the ultrastructural level in experimental NMOSD lesions, EM was performed. Animals were deeply anesthetized and perfused first with PBS and subsequently with $4 \%$ PFA. The brain was removed and cut coronally at the injection site. The area around the injection site was dissected into $1 \mathrm{~mm}^{2}$ pieces and postfixed in $3 \%$ glutaraldehyde overnight. The tissue was then incubated with $1 \%$ osmium tetroxide solution for 1 hour at $4^{\circ} \mathrm{C}$, dehydrated in $50 \%$ ethanol, and additionally stained with $0.5 \%$ uranyl acetate for 1 hour at $4^{\circ} \mathrm{C}$. After further dehydration (in 70, 80, 96, and $100 \%$ ethanol, propylene oxide), propylene oxide was replaced stepwise by Renlam M1 resin (Serva).

Resin blocks were cut in semithin sections of 360-400 nm and stained for 3 minutes with Richardson's stain, which stains myelin in dark blue and cytoplasm in light blue. The area of interest was trimmed, and ultrathin sections of 70 to $72 \mathrm{~nm}$ were cut and then stained with lead citrate for 10 minutes and imaged using the electron microscope EM1OB (Zeiss).

The formalin-fixed human NMOSD spinal cord tissue was embedded in $20 \%$ gelatin on ice. Sections of $200 \mu \mathrm{m}$ thickness were then made using a Leica Vibratome VT1200S (Leica) and post-fixed for 3 days in $2.5 \%$ glutaraldehyde and $4 \%$ paraformaldehyde in phosphate buffer according to Karlsson-Schultz (83). The tissue was embedded for EM in Epon (Serva) after post-fixation with $2 \% \mathrm{OsO}_{4}$ (Science Services) and then dehydrated with ethanol and propylene oxide using an automated system (EMTP Leica Microsystems) (83). Ultrathin sections of the Epon-embedded samples were cut at $50 \mathrm{~nm}$ thickness and imaged using a Zeiss EM 900 or LEO912 AB electron microscope (Zeiss) equipped with a 2k CCD camera (TRS).
Statistics. To compare 2 groups, the independent 2-sided $t$ test with Welch's correction was used for parametric data and the 2-sided Mann-Whitney $U$ test for nonparametric data. To compare more than 2 groups, the Kruskal-Wallis test followed by Dunn's multiple comparison test was performed. Pearson's correlation coefficients were determined to assess associations between the area of FITC-albumin extravasation and the number of infiltrated PMNs. Statistical significance was defined as $P<0.05$. All data are given as mean \pm SEM. Statistical analysis was carried out using GraphPad Prism software, version 5.01.

Study approval. Human tissue was obtained from the archives of the Institute of Neuropathology at the University Medical Centre Göttingen. The study was approved by the local ethics committee. All animal experiments were conducted in accordance with the European Communities Council Directive of September 22, 2010 (2010/63/EU) and were approved by the Lower Saxony State Office for Consumer Protection and Food Safety, Germany.

\section{Author contributions}

AW and CS conceived the project. AW, CW, MH, MTW, WM, and SN performed experiments. WM, FO, DRT, MC, GO, MG, and JLB provided resources. AW, MH, and SN analyzed data. AW and CS wrote the original draft of the manuscript. AW, CW, MH, MTW, WM, FO, DRT, MC, GO, JLB, SN, and CS reviewed and edited the manuscript. CS acquired funding. CS supervised the study.

\section{Acknowledgments}

This project was supported by the Deutsche Forschungsgemeinschaft (DFG) (STA 1389/2-1 and CRCs 43 and 274, 408885537), the DFG under Germany's Excellence Strategy (EXC 2067/1390729940), the Gemeinnützige Hertie Foundation, the Deutsche Multiple Sklerose Gesellschaft (DMSG), and the National MS Society (USA) (to CS). MTW was supported by the Cluster of Excellence and DFG Research CNMPB, Göttingen, Germany. JLB received grants from the Guthy Jackson Charitable Foundation and the NIH (R01EY022936). MC was supported by the American Diabetes Association Pathway to Stop Diabetes (1-15-ACN-06), and GO was supported by the Research Foundation of Flanders (7516N and 5716N) and C1 funding by KU Leuven. We acknowledge the excellent technical support of Heidi Brodmerkel, Angela Dettmar, Olga Kowatsch, Brigitte Maruschak, Jasmin Reichl, and Katja Schulz and thank Cynthia Bunker for language editing.

Address correspondence to: Christine Stadelmann, Institute of Neuropathology, Robert-Koch-Strasse 40, 37099 Göttingen, Germany. Phone:49.551.39.67570; Email: cstadelmann@med. uni-goettingen.de.

\section{CW's present address is: Evotec, Göttingen, Germany.}

MTW's present address is: AbbVie, Ludwigshafen am Rhein, Germany.
1. Flanagan EP, et al. Epidemiology of aquaporin-4 autoimmunity and neuromyelitis optica spectrum. Ann Neurol. 2016;79(5):775-783.

2. Wrzos C, et al. Early loss of oligodendrocytes in human and experimental neuromyelitis optica lesions. Acta Neuropathol. 2014;127(4):523-538.

3. Papadopoulos M, Verkman A. Aquaporin 4 and neuromyelitis optica. Lancet Neurol. 2012;11(6):535-544.

4. Bennett J, et al. Intrathecal pathogenic anti-aqua- porin-4 antibodies in early neuromyelitis optica. Ann Neurol. 2009;66(5):617-629.

5. Bradl M, et al. Neuromyelitis optica: pathogenicity of patient immunoglobulin in vivo. Ann Neurol. 2009;66(5):630-643. 
6. Kinoshita M, et al. Neuromyelitis optica: passive transfer to rats by human immunoglobulin. Biochem Biophys Res Commun. 2009;386(4):623-627.

7. Ratelade J, et al. Involvement of antibody-dependent cell-mediated cytotoxicity in inflammatory demyelination in a mouse model of neuromyelitis optica. Acta Neuropathol. 2013;126(5):699-709.

8. Saadoun S, et al. Intra-cerebral injection of neuromyelitis optica immunoglobulin $\mathrm{G}$ and human complement produces neuromyelitis optica lesions in mice. Brain. 2010;133(Pt 2):349-361.

9. Pittock S, et al. Eculizumab in AQP4-IgG-positive relapsing neuromyelitis optica spectrum disorders: an open-label pilot study. Lancet Neurol. 2013;12(6):554-562.

10. Papadopoulos MC, et al. Treatment of neuromyelitis optica: state-of-the-art and emerging therapies. Nat Rev Neurol. 2014;10(9):493-506.

11. Kitic M, et al. Intrastriatal injection of interleukin- 1 beta triggers the formation of neuromyelitis optica-like lesions in NMO-IgG seropositive rats. Acta Neuropathol Commun. 2013;1:5.

12. Ratelade J, et al. Intravenous neuromyelitis optica autoantibody in mice targets aquaporin- 4 in peripheral organs and area postrema. PLoS One. 2011;6(11):e27412.

13. Hillebrand S, et al. Circulating AQP4-specific auto-antibodies alone can induce neuromyelitis optica spectrum disorder in the rat. Acta Neuropathol. 2019;137(3):467-485.

14. Nishiyama S, et al. A case of NMO seropositive for aquaporin- 4 antibody more than 10 years before onset. Neurology. 2009;72(22):1960-1961.

15. Araki M, et al. Efficacy of the anti-IL-6 receptor antibody tocilizumab in neuromyelitis optica: a pilot study. Neurology. 2014;82(15):1302-1306.

16. Ringelstein $\mathrm{M}$, et al. Long-term therapy with interleukin 6 receptor blockade in highly active neuromyelitis optica spectrum disorder. JAMA Neurol. 2015;72(7):756-763.

17. Yamamura T, et al. Trial of satralizumab in neuromyelitis optica spectrum disorder. N Engl J Med. 2019;381(22):2114-2124.

18. Uzawa A, et al. Markedly elevated soluble intercellular adhesion molecule 1 , soluble vascular cell adhesion molecule 1 levels, and blood-brain barrier breakdown in neuromyelitis optica. Arch Neurol. 2011;68(7):913-917.

19. Rochfort KD, Cummins PM. Cytokine-mediated dysregulation of zonula occludens-1 properties in human brain microvascular endothelium. Microvasc Res. 2015;100:48-53.

20. Takeshita Y, et al. Effects of neuromyelitis optica-IgG at the blood-brain barrier in vitro. Neurol Neuroimmunol Neuroinflamm. 2017;4(1):e311.

21. Shimizu F, et al. Glucose-regulated protein 78 autoantibody associates with blood-brain barrier disruption in neuromyelitis optica. Sci Transl Med. 2017;9(397):eaai9111.

22. Tomizawa Y, et al. Blood-brain barrier disruption is more severe in neuromyelitis optica than in multiple sclerosis and correlates with clinical disability. J Int Med Res. 2012;40(4):1483-1491.

23. Obermeier B, et al. Development, maintenance and disruption of the blood-brain barrier. Nat Med. 2013;19(12):1584-1596.

24. Larochelle C, et al. How do immune cells overcome the blood-brain barrier in multiple sclero- sis? FEBS Lett. 2011;585(23):3770-3780.

25. Lucchinetti CF, et al. A role for humoral mechanisms in the pathogenesis of Devic's neuromyelitis optica. Brain. 2002;125(Pt 7):1450-1461.

26. Kirk J, et al. Tight junctional abnormality in multiple sclerosis white matter affects all calibres of vessel and is associated with blood-brain barrier leakage and active demyelination. J Pathol. 2003;201(2):319-327.

27. Plumb J, et al. Abnormal endothelial tight junctions in active lesions and normal-appearing white matter in multiple sclerosis. Brain Pathol. 2002;12(2):154-169.

28. Liebner S, et al. Claudin-1 and claudin-5 expression and tight junction morphology are altered in blood vessels of human glioblastoma multiforme. Acta Neuropathol. 2000;100(3):323-331.

29. Wolburg $\mathrm{H}$, et al. Localization of claudin-3 in tight junctions of the blood-brain barrier is selectively lost during experimental autoimmune encephalomyelitis and human glioblastoma multiforme. Acta Neuropathol. 2003;105(6):586-592.

30. Luissint AC, et al. Tight junctions at the blood brain barrier: physiological architecture and disease-associated dysregulation. Fluids Barriers CNS. 2012;9(1):23.

31. Carlson T, et al. The Th17-ELR+ CXC chemokine pathway is essential for the development of central nervous system autoimmune disease. J Exp Med. 2008;205(4):811-823.

32. Morrissey SP, et al. In vivo mri and its histologica correlates in acute adoptive transfer experimental allergic encephalomyelitis. Quantification of inflammation and oedema. Brain. 1996;119(Pt 1):239-248.

33. Zhang H, et al. Ex vivo spinal cord slice model of neuromyelitis optica reveals novel immunopathogenic mechanisms. Ann Neurol. 2011;70(6):943-954.

34. Chen F, et al. Disruptions of occludin and claudin-5 in brain endothelial cells in vitro and in brains of mice with acute liver failure. Hepatology. 2009;50(6):1914-1923.

35. Suzuki N, et al. Mechanism for the increased permeability in endothelial monolayers induced by elastase. Mediators Inflamm. 1994;3(1):11-16.

36. Gao M, et al. Acceleration of diabetic wound healing using a novel protease-anti-protease combination therapy. Proc Natl Acad Sci US A. 2015;112(49):15226-15231.

37. Champagne B, et al. Proteolytic cleavage of ICAM-1 by human neutrophil elastase. J Immunol.1998;161(11):6398-6405.

38. Kaynar AM, et al. Neutrophil elastase is needed for neutrophil emigration into lungs in ventilator-induced lung injury. Am J Respir Cell Mol Biol. 2008;39(1):53-60.

39. Stewart PA, Wiley MJ. Developing nervous tissue induces formation of blood-brain barrier characteristics in invading endothelial cells: a study using quail-chick transplantation chimeras. Dev Biol. 1981;84(1):183-192.

40. Abbott NJ, et al. Astrocyte-endothelial interactions at the blood-brain barrier. Nat Rev Neurosci. 2006;7(1):41-53.

41. Bush TG, et al. Leukocyte infiltration, neuronal degeneration, and neurite outgrowth after ablation of scar-forming, reactive astrocytes in adult transgenic mice. Neuron. 1999;23(2):297-308.

42. Tognatta $\mathrm{R}$, et al. Astrocytes are required for oligodendrocyte survival and maintenance of myelin compaction and integrity. Front Cell Neurosci. 2020;14:74

43. Willis CL, et al. Focal astrocyte loss is followed by microvascular damage, with subsequent repair of the blood-brain barrier in the apparent absence of direct astrocytic contact. Glia. 2004;45(4):325-337.

44. Willis CL, et al. Partial recovery of the damaged rat blood-brain barrier is mediated by adherens junction complexes, extracellular matrix remodeling and macrophage infiltration following focal astrocyte loss. Neuroscience. 2013;250:773-785.

45. Krum JM, Rosenstein JM. Effect of astroglial degeneration on the blood-brain barrier to protein in neonatal rats. Brain Res Dev Brain Res. 1993;74(1):41-50.

46. Krum JM. Experimental gliopathy in the adult rat CNS: effect on the blood-spinal cord barrier. Glia. 1994;11(4):354-366.

47. Adams RA, et al. The fibrin-derived gamma377395 peptide inhibits microglia activation and suppresses relapsing paralysis in central nervous system autoimmune disease. J Exp Med 2007;204(3):571-582

48. Ryu JK, et al. Blood coagulation protein fibrinogen promotes autoimmunity and demyelination via chemokine release and antigen presentation. Nat Commun. 2015;6:8164

49. Lee NJ, et al. Spatiotemporal distribution of fibrinogen in marmoset and human inflammatory demyelination. Brain. 2018;141(6):1637-1649.

50. Marik C, et al. Lesion genesis in a subset of patients with multiple sclerosis: a role for innate immunity? Brain. 2007;130(Pt 11):2800-2815.

51. Nitta T, et al. Size-selective loosening of the blood-brain barrier in claudin-5-deficient mice. J Cell Biol. 2003;161(3):653-660.

52. Kooij G, et al. Disturbed function of the blood-cerebrospinal fluid barrier aggravates neuro-inflammation. Acta Neuropathol. 2014;128(2):267-277.

53. Saitou M, et al. Complex phenotype of mice lacking occludin, a component of tight junction strands. Mol Biol Cell. 2000;11(12):4131-4142.

54 . Furuse M, et al. A single gene product, claudin-1 or -2 , reconstitutes tight junction strands and recruits occludin in fibroblasts. JCell Biol. 1998;143(2):391-401.

55. Morita K, et al. Endothelial claudin: claudin-5/ TMVCF constitutes tight junction strands in endothelial cells. J Cell Biol. 1999;147(1):185-194.

56. Argaw AT, et al. VEGF-mediated disruption of endothelial CLN-5 promotes blood-brain barrier breakdown. Proc Natl Acad Sci U S A. 2009;106(6):1977-1982.

57. Halder SK, et al. Hypoxic pre-conditioning suppresses experimental autoimmune encephalomyelitis by modifying multiple properties of blood vessels. Acta Neuropathol Commun 2018;6(1):86

58. Lutz SE, et al. Caveolin1 is required for Th1 cell infiltration, but not tight junction remodeling, at the blood-brain barrier in autoimmune neuroinflammation. Cell Rep. 2017;21(8):2104-2117.

59. Ferrara G, et al. NG2, a common denominator for 
neuroinflammation, blood-brain barrier alteration, and oligodendrocyte precursor response in EAE, plays a role in dendritic cell activation. Acta Neuropathol. 2016;132(1):23-42.

60. von Wedel-Parlow M, et al. Neutrophils cross the BBB primarily on transcellular pathways: an in vitro study. Brain Res. 2011;1367:62-76.

61. Armulik A, et al. Pericytes regulate the bloodbrain barrier. Nature. 2010;468(7323):557-561.

62. Bell RD, et al. Pericytes control key neurovascular functions and neuronal phenotype in the adult brain and during brain aging. Neuron . 2010;68(3):409-427.

63. Saadoun S, et al. T cell deficiency does not reduce lesions in mice produced by intracerebral injection of NMO-IgG and complement. J Neuroimmunol. 2011;235(1-2):27-32.

64. Saadoun S, et al. Neutrophil protease inhibition reduces NMO-IgG-induced damage in mouse brain. Ann Neurol. 2012;71(3):323-333.

65. Zhang H, Verkman AS. Eosinophil pathogenicity mechanisms and therapeutics in neuromyelitis optica. J Clin Invest. 2013;123(5):2306-2316.

66. Schnell L, et al. Cytokine-induced acute inflammation in the brain and spinal cord. JNeuropathol Exp Neurol. 1999;58(3):245-254.

67. Bolton SJ, et al. Loss of the tight junction proteins occludin and zonula occludens- 1 from cerebral vascular endothelium during neutrophil-induced blood-brain barrier breakdown in vivo. Neuroscience. 1998;86(4):1245-1257.
68. Ferrari CC, et al. Reversible demyelination, blood-brain barrier breakdown, and pronounced neutrophil recruitment induced by chronic IL-1 expression in the brain. Am J Pathol. 2004;165(5):1827-1837.

69. Aube B, et al. Neutrophils mediate blood-spinal cord barrier disruption in demyelinating neuroinflammatory diseases. JImmunol. 2014;193(5):2438-2454.

70. Gijbels K, et al. Gelatinase B is present in the cerebrospinal fluid during experimental autoimmune encephalomyelitis and cleaves myelin basic protein. J Neurosci Res. 1993;36(4):432-440.

71. Ehrengruber MU, et al. Activation of human neutrophils by C3a and C5A. Comparison of the effects on shape changes, chemotaxis, secretion, and respiratory burst. FEBS Lett. 1994;346(2-3):181-184.

72. Piatek P, et al. C5a-preactivated neutrophils are critical for autoimmune-induced astrocyte dysregulation in neuromyelitis optica spectrum disorder. Front Immunol. 2018;9:1694.

73. Wolpe SD, et al. Identification and characterization of macrophage inflammatory protein 2. Proc Natl Acad Sci U S A. 1989;86(2):612-616.

74. Yao J, et al. Chemotaxis by a CNS macrophage, the microglia. J Neurosci Res. 1990;27(1):36-42.

75. Chen T, et al. Astrocyte-microglia interaction drives evolving neuromyelitis optica lesion. JClin Invest. 2020;130(8):4025-4038.

76. DiStasi MR, Ley K. Opening the flood-gates: how neutrophil-endothelial interactions regulate permeability. Trends Immunol. 2009;30(11):547-556.

77. Segel GB, et al. The paradox of the neutrophil's role in tissue injury. J Leukoc Biol. 2011;89(3):359-372.

78. Wingerchuk DM, et al. International consensus diagnostic criteria for neuromyelitis optica spectrum disorders. Neurology. 2015;85(2):177-189.

79. Sekiya S, et al. Selective depletion of rat neutrophils by in vivo administration of a monoclonal antibody. J Leukoc Biol. 1989;46(2):96-102.

80. Schneider CA, et al. NIH Image to ImageJ: 25 years of image analysis. Nat Methods. 2012;9(7):671-675.

81. Abbott NJ, et al. An improved in vitro bloodbrain barrier model: rat brain endothelial cells co-cultured with astrocytes. Methods Mol Biol. 2012;814:415-430.

82. Perriere N, et al. Puromycin-based purification of rat brain capillary endothelial cell cultures. Effect on the expression of blood-brain barrier-specific properties. J Neurochem. 2005;93(2):279-289.

83. Mobius W, et al. Electron microscopy of the mouse central nervous system. Methods Cell Biol. 2010;96:475-512.

84. Radzun HJ, et al. Detection of a monocyte/ macrophage differentiation antigen in routinely processed paraffin-embedded tissues by monoclonal antibody Ki-M1P. Lab Invest. 1991;65(3):306-315. 